\title{
Viscoelastic fluids in thin domains: a mathematical proof
}

\author{
Guy Bayada ${ }^{1,2}$, Laurent Chupin ${ }^{2}$ and Bérénice Grec ${ }^{3, *}$ \\ Batiment Léonard de Vinci - 21, avenue Jean Capelle \\ 69621 Villeurbanne cedex - France
}

\begin{abstract}
The present paper deals with non Newtonian viscoelastic flows of Oldroyd-B type in thin domains. Such geometries arise for example in the context of lubrication. More precisely, we justify rigorously the asymptotic model obtained heuristically by proving the mathematical convergence of the Navier-Stokes/Oldroyd-B sytem towards the asymptotic model.
\end{abstract}

Keywords: Viscoelastic fluids, Thin film, Oldroyd model, Lubrication flow, Asymptotic analysis.

\section{Introduction}

This paper concerns the study of a viscoelastic fluid flow in a thin gap, the motion of which is imposed due to non homogeneous boundary conditions.

When a Newtonian flow is contained between two close given surfaces in relative motion, it is well known that it is possible to replace the Stokes or Navier-Stokes equations governing the fluid's motion by a simpler asymptotic model. The asymptotic pressure is proved to be independent of the normal direction to the close surfaces and obeys the Reynolds thin film equation whose coefficients include the velocities, the geometrical description of the surrounding surfaces and some rheological characteristics of the fluid. As a following step, the computation of this pressure allows an asymptotic velocity of the fluid to be easily computed. Such asymptotic procedure first proposed in a formal way by Reynolds [2] has been rigorously confirmed for Newtonian stationary flow [1], and then generalized in a lot of situations covering numerous applications for both compressible fluid [14], unsteady cases [3], multifluid flows [15].

It is well known however that in numerous applications, the fluid to be considered is a non Newtonian one. This is the case for numerous biological fluids, modern lubricants in engineering applications due to the additives they contain, polymers in injection or molding process. In all of these applications, there are situations in which the flow is anisotropic. It is usual to take account of this geometrical effect in order to simplify the three-dimensional equations of the motion,

\footnotetext{
${ }^{1}$ INSA-Lyon - Institut Camille Jordan - CNRS UMR 5208

${ }^{2}$ INSA-Lyon - LAMCOS - CNRS UMR 5259

${ }^{3}$ Ecole Centrale de Lyon - Institut Camille Jordan - CNRS UMR 5208

*E-mail: berenice.grec@insa-lyon.fr, Tel: +33 4724370 40, Fax: +3347243 4529 .
} 
trying to recover two dimensional Reynolds like equation with respect to the pressure only. Such procedures are more often heuristic ones. Nevertheless, some mathematical works appeared in the literature to justify them. They include thin film asymptotic studies of Bingham flow [9], quasi Newtonian flow (Carreau's law, power law or Williamson's law, in which various stress-velocity relations are chosen: [7], [6], [16]) and also micro polar ones [5]. It has been possible to obtain rigorously some thin film approximation for such fluids using a so called generalized Reynolds equation for the pressure.

However in the preceding examples, elasticity effects are neglected. Introduction of such viscoelastic behavior is characterized by the Deborah number which is related to the relaxation time. One of the most popular laws is the Oldroyd-B model whose constitutive equation is an interpolation between purely viscous and purely elastic models, thus introducing an additional parameter which describes the relative proportion of both behaviors. A formal procedure has been proposed in [4]. However, the asymptotic system so obtained lacks the usual characteristic of classical generalized Reynolds equation as it has not been possible to gain an equation in the asymptotic pressure only. Both velocity $u^{*}$ and pressure $p^{*}$ are coupled by a non linear system.

It is the goal of this paper to justify rigorously this asymptotic system. Section 2 is devoted to the precise statement of the 3-D problem. One difficulty has been to find an existence theorem for the general Oldroyd-B model, acting as a starting point for the mathematical procedure. Most of the existence theorems, however, deal with small data or small time assumptions. To control this kind of property with respect to the smallness of the gap appears somewhat difficult. So we are led to consider a more particular Oldroyd-B model, for which unconditional existence theorem has been proved [13]. Moreover, a specific attention is devoted to the boundary conditions to be introduced both on the velocity and on the stress. The goal is to use "well prepared" boundary conditions so as to prevent boundary layer on the lateral side of the domain.

In Section 3, after suitable scaling procedure, asymptotic expansions of both pressure, viscosity and stress are introduced, taking into account the previous formal results from [4]. Section 4 is mainly concerned with the proof of some additional regularity properties for the formal asymptotic solution. Assuming some restrictions on the rheological parameters, it will be proved that it is possible to gain a $C^{k}$ regularity for $p^{*}, k>1$, which in turn improves the regularity of $u^{*}$ and the stress tensor $\sigma^{*}$. This result is obtained by introducing a differential Cauchy system satisfied by the derivative of $p^{*}$. Finally, section 5, is devoted to the convergence towards zero of the second term of the asymptotic expansions, which in turn proves the convergence of the solution of the real 3-D problem towards $u^{*}, p^{*}, \sigma^{*}$ (Theorems 5.4 and 5.6).

\section{Introduction of the problem and known results}

\subsection{Formulation of the problem}

We consider unsteady incompressible flows of viscoelastic fluids, which are ruled by Oldroyd's law, in a thin domain $\hat{\Omega}^{\varepsilon}=\left\{(x, y) \in \mathbb{R}^{n}, x \in \omega\right.$ and $\left.0<y<\varepsilon h(x)\right\}$, where $\omega$ is an $(n-1)$-dimensional domain, with $n=2$ or $n=3\left(x=x_{1}\right.$ or $\left.x=\left(x_{1}, x_{2}\right)\right)$, as in Figure 1. 


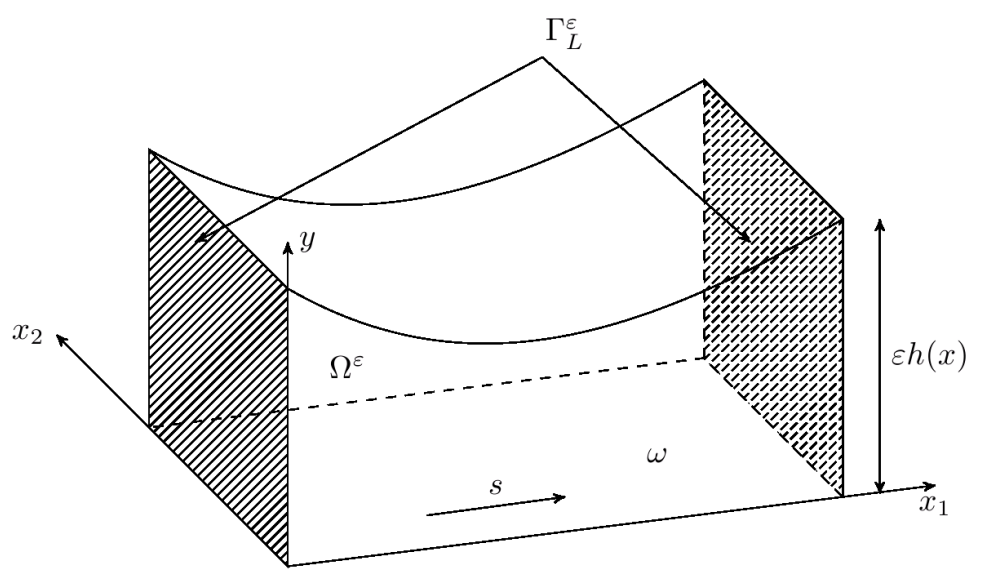

Figure 1: Domain $\hat{\Omega}_{\varepsilon}$

The following hypotheses on $h$ are required:

$$
\forall x \in \omega, 0<h_{0} \leq h(x) \leq h_{M}^{\varepsilon}, \quad \text { and } \quad h^{\varepsilon} \in \mathcal{C}^{1}(\bar{\omega})
$$

Let $\hat{\boldsymbol{u}}^{\varepsilon}=\left(\hat{u}_{1}^{\varepsilon}, \hat{u}_{2}^{\varepsilon}, \hat{u}_{3}^{\varepsilon}\right)$ be the velocity field in the three-dimensional case, or $\hat{\boldsymbol{u}}^{\varepsilon}=\left(\hat{u}_{1}^{\varepsilon}, \hat{u}_{2}^{\varepsilon}\right)$ in the two-dimensional case, $\hat{p}^{\varepsilon}$ the pressure, and $\hat{\boldsymbol{\sigma}}^{\varepsilon}$ the stress symmetric tensor in the domain $\hat{\Omega}^{\varepsilon}$. Bold letters stand for vectorial or tensorial functions, the notation $\hat{f}$ corresponds to a function $f$ defined in the domain $\hat{\Omega}^{\varepsilon}$, and the superscript ${ }^{\varepsilon}$ denotes the dependence on $\varepsilon$.

Formulation of the problem The following formulation of the problem holds in $(0, \infty) \times \hat{\Omega}^{\varepsilon}$ :

$$
\left\{\begin{aligned}
\rho \partial_{t} \hat{\boldsymbol{u}}^{\varepsilon}+\rho \hat{\boldsymbol{u}}^{\varepsilon} \cdot \nabla \hat{\boldsymbol{u}}^{\varepsilon}-(1-r) \nu \Delta \hat{\boldsymbol{u}}^{\varepsilon}+\nabla \hat{p}^{\varepsilon} & =\nabla \cdot \hat{\boldsymbol{\sigma}}^{\varepsilon}, \\
\nabla \cdot \hat{\boldsymbol{u}}^{\varepsilon} & =0 \\
\lambda\left(\partial_{t} \hat{\boldsymbol{\sigma}}^{\varepsilon}+\hat{\boldsymbol{u}}^{\varepsilon} \cdot \nabla \hat{\boldsymbol{\sigma}}^{\varepsilon}+g\left(\hat{\boldsymbol{\sigma}}^{\varepsilon}, \nabla \hat{\boldsymbol{u}}^{\varepsilon}\right)\right)+\hat{\boldsymbol{\sigma}}^{\varepsilon} & =2 r \nu D\left(\hat{\boldsymbol{u}}^{\varepsilon}\right),
\end{aligned}\right.
$$

where the nonlinear terms $g\left(\hat{\boldsymbol{\sigma}}^{\varepsilon}, \nabla \hat{\boldsymbol{u}}^{\varepsilon}\right)$, the vorticity tensor $W\left(\hat{\boldsymbol{u}}^{\varepsilon}\right)$ and the deformation tensor $D\left(\hat{\boldsymbol{u}}^{\varepsilon}\right)$ are given by:

$$
\begin{gathered}
g\left(\hat{\boldsymbol{\sigma}}^{\varepsilon}, \nabla \hat{\boldsymbol{u}}^{\varepsilon}\right)=-W\left(\hat{\boldsymbol{u}}^{\varepsilon}\right) \cdot \hat{\boldsymbol{\sigma}}^{\varepsilon}+\hat{\boldsymbol{\sigma}}^{\varepsilon} \cdot W\left(\hat{\boldsymbol{u}}^{\varepsilon}\right), \\
W\left(\hat{\boldsymbol{u}}^{\varepsilon}\right)=\frac{\nabla \hat{\boldsymbol{u}}^{\varepsilon}-{ }^{t} \nabla \hat{\boldsymbol{u}}^{\varepsilon}}{2} \quad \text { and } \quad D\left(\hat{\boldsymbol{u}}^{\varepsilon}\right)=\frac{\nabla \hat{\boldsymbol{u}}^{\varepsilon}+{ }^{t} \nabla \hat{\boldsymbol{u}}^{\varepsilon}}{2} .
\end{gathered}
$$

In this formulation, the physical parameters are the viscosity $\nu$, the density $\rho$, and the relaxation time $\lambda$. The parameter $\lambda$ is related to the viscoelastic behavior and the Deborah number. The parameter $r \in[0,1)$ describes the relative proportion of the viscous and elastic behavior. 
Initial conditions This problem is considered with the following initial conditions:

$$
\left.\hat{\boldsymbol{u}}^{\varepsilon}\right|_{t=0}=\hat{\boldsymbol{u}}_{0}^{\varepsilon},\left.\quad \hat{\boldsymbol{\sigma}}^{\varepsilon}\right|_{t=0}=\hat{\boldsymbol{\sigma}}_{0}^{\varepsilon}
$$

for $\hat{\boldsymbol{u}}_{0}^{\varepsilon} \in \boldsymbol{L}^{2}\left(\hat{\Omega}^{\varepsilon}\right), \hat{\boldsymbol{\sigma}}_{0}^{\varepsilon} \in \boldsymbol{L}^{2}\left(\hat{\Omega}^{\varepsilon}\right)$. The bold notation $\boldsymbol{L}^{2}\left(\hat{\Omega}^{\varepsilon}\right)$ denotes the set of vectorial or tensorial functions whose all components belong to $L^{2}\left(\hat{\Omega}^{\varepsilon}\right)$.

Boundary conditions Dirichlet boundary conditions are set on top and bottom of the domain, and the conditions on the lateral part of the boundary $\hat{\Gamma}_{L}^{\varepsilon}$, defined by

$$
\hat{\Gamma}_{L}^{\varepsilon}=\left\{(x, y) \in \mathbb{R}^{n}, x \in \partial \omega \text { and } 0<y<\varepsilon h(x)\right\}
$$

will be specified later (in section 4.2). Therefore, it is possible to write the boundary conditions in a shortened way:

$$
\left.\hat{\boldsymbol{u}}^{\varepsilon}\right|_{\partial \hat{\Omega}^{\varepsilon}}=\hat{\boldsymbol{J}}^{\varepsilon}
$$

where $\hat{\boldsymbol{J}}^{\varepsilon}$ is a given function such that $\hat{\boldsymbol{J}}^{\varepsilon} \in \boldsymbol{H}^{1 / 2}\left(\partial \hat{\Omega}^{\varepsilon}\right)$ and satisfying $\left.\hat{\boldsymbol{J}}^{\varepsilon}\right|_{y=h^{\varepsilon}}=0,\left.\hat{\boldsymbol{J}}^{\varepsilon}\right|_{y=0}=(s, 0)$. This function will be fully determined in Subsection 4.2 .

Since $\hat{\boldsymbol{\sigma}}^{\varepsilon}$ satisfies a transport equation in the domain $\hat{\Omega}^{\varepsilon}$, it remains to impose boundary conditions on $\hat{\boldsymbol{\sigma}}^{\varepsilon}$ on the part of the boundary where $\hat{\boldsymbol{u}}^{\varepsilon}$ is an incoming velocity. Let us define $\hat{\Gamma}_{+}^{\varepsilon}$ the part of $\hat{\Gamma}_{L}^{\varepsilon}$ such that $\left.\hat{\boldsymbol{J}}^{\varepsilon}\right|_{\hat{\Gamma}_{+}^{\varepsilon}} \cdot n<0$, and $\hat{\Gamma}_{-}^{\varepsilon}=\hat{\Gamma}_{L}^{\varepsilon} \backslash \hat{\Gamma}_{+}^{\varepsilon}$. We set

$$
\left.\hat{\boldsymbol{\sigma}}^{\varepsilon}\right|_{\hat{\Gamma}_{+}^{\varepsilon}}=\hat{\boldsymbol{\theta}}^{\varepsilon}
$$

where $\hat{\boldsymbol{\theta}}^{\varepsilon}$ is a given function in $\boldsymbol{H}^{1 / 2}\left(\hat{\Gamma}_{+}^{\varepsilon}\right)$ which will also be determined in Subsection 4.2.

Moreover, since the pressure is defined up to a constant, the mean pressure is chosen to be zero: $\int_{\hat{\Omega}^{\varepsilon}} \hat{p}^{\varepsilon}=0$.

Notations Let us introduce the following function space:

$$
V=\left\{\hat{\boldsymbol{\varphi}} \in \boldsymbol{H}_{0}^{1}\left(\hat{\Omega}^{\varepsilon}\right), \nabla \cdot \hat{\boldsymbol{\varphi}}=0\right\}
$$

and the following notations, that will be used in the following. For $\hat{f}$ defined in $\hat{\Omega}^{\varepsilon}$ :

- $|\hat{f}|$ denotes the $L^{2}$-norm in $\hat{\Omega}^{\varepsilon}$,

- $|\hat{f}|_{p}$ denotes the $L^{p}$-norm in $\hat{\Omega}^{\varepsilon}$, for $2<p \leq+\infty$,

- the spaces $\mathcal{C}^{m}\left(\overline{\hat{\Omega}^{\varepsilon}}\right)$ for $m \geq 1$ are equipped with the norms $\|\hat{f}\|_{\mathcal{C}^{m}}=|\hat{f}|_{\infty}+\sum_{i=1}^{m}\left|\hat{f}^{(i)}\right|_{\infty}$.

For $\hat{f}$ defined in $\mathbb{R}^{+} \times \hat{\Omega}^{\varepsilon},\|\hat{f}\|_{L^{\alpha}\left(L^{\beta}\right)}$ denotes the norm of the space $L^{\alpha}\left(0, \infty, L^{\beta}\left(\hat{\Omega}^{\varepsilon}\right)\right)$, with $1 \leq$ $\alpha, \beta \leq \infty$. 


\subsection{Existence theorem in the domain $\hat{\Omega}^{\varepsilon}$}

Theorem 2.1. For $\varepsilon>0$ fixed, problem (2.1)-(2.3) admits a weak solution

$$
\hat{\boldsymbol{u}}^{\varepsilon} \in L_{l o c}^{2}\left(0, \infty, \boldsymbol{H}^{1}\left(\hat{\Omega}^{\varepsilon}\right)\right), \quad \hat{p}^{\varepsilon} \in L_{l o c}^{2}\left(0, \infty, L^{2}\left(\hat{\Omega}^{\varepsilon}\right)\right), \quad \hat{\boldsymbol{\sigma}}^{\varepsilon} \in \mathcal{C}\left(0, \infty, \boldsymbol{L}^{2}\left(\hat{\Omega}^{\varepsilon}\right)\right) .
$$

Proof. This result is proved in [13].

Remark 2.2. Let us emphasize that for the following, it is essential to know the global (in time) existence of a solution for problem (2.1)-(2.3). Other existence theorems have been proved for this problem, for example in [12], [11], [10], but these theorems are either local in time (on a time interval $\left.\left[0, T^{\varepsilon}\right]\right)$, or a small data assumption is needed. In this work, these theorems cannot be used, since there is no control on the behavior of $T^{\varepsilon}$ (or equivalently of the data) when $\varepsilon$ tends to zero, in particular $T^{\varepsilon}$ may tend to zero.

Consequently, this work is restricted to the specific case treated in [13], taking one parameter of the Oldroyd model to be zero. In all generality, the non-linear term reads $g(\boldsymbol{\sigma}, \nabla \boldsymbol{u})=-W(\boldsymbol{u})$. $\boldsymbol{\sigma}+\boldsymbol{\sigma} \cdot W(\boldsymbol{u})-a(\boldsymbol{\sigma} \cdot D(\boldsymbol{u})+D(\boldsymbol{u}) \cdot \boldsymbol{\sigma})$, which is called objective derivative. Here the parameter a is taken to be zero. This case corresponds to the so-called Jaumann derivative.

Remark 2.3. The following computations are made in the two-dimensional case (i.e. $\omega=(0, L)$ is a one-dimensional domain) for the sake of simplicity. However, note that except for the regularity obtained for the limit problem in Section 4.3, all estimates are independent of the dimension, thus the corresponding computations should apply to the three-dimensional case.

Regularizing the system In the proof of the preceding theorem, the existence of a solution is achieved by regularization. Therefore, this study only concerns solutions obtained as the limit of a regularized problem approximating (2.1), in which an additional term $-\eta \Delta \hat{\boldsymbol{\sigma}}^{\varepsilon \eta}$ is added to the Oldroyd equation, with $\eta>0$ a small parameter. Here a regularization of the form $-\eta \Delta\left(\hat{\boldsymbol{\sigma}}^{\varepsilon \eta}-\hat{\boldsymbol{G}}\right)$ is chosen, with $\hat{\boldsymbol{G}}$ a symmetric tensor in $\boldsymbol{H}^{2}\left(\hat{\Omega}^{\varepsilon}\right)$ independent of $\eta$ and $\varepsilon$ which will be precised later. After obtaining the needed energy estimates uniformly in $\eta$, we will let $\eta$ tend to zero. This approach allows to multiply the Oldroyd equation by $\hat{\boldsymbol{\sigma}}^{\varepsilon \eta}$, since $\hat{\boldsymbol{\sigma}}^{\varepsilon \eta}$ is regular enough. Of course, one can choose another regularization which leads to energy estimates which are uniform in the regularization parameter.

Furthermore, because of the regularizing term, boundary conditions on the whole boundary are needed. Let us write $\left.\hat{\boldsymbol{\sigma}}^{\varepsilon \eta}\right|_{\partial \hat{\Omega}^{\varepsilon}}=\hat{\boldsymbol{\theta}}^{\varepsilon \eta}$, where $\hat{\boldsymbol{\theta}}^{\varepsilon \eta}$ is now a function of $\boldsymbol{H}^{1 / 2}\left(\partial \hat{\Omega}^{\varepsilon}\right)$, which will be determined later by equation (4.3). 


\section{Asymptotic expansions}

\subsection{Renormalization of the domain}

Introducing a new variable $z=\frac{y}{\varepsilon}$, the system (2.1) can be rewritten in a fixed re-scaled domain:

$$
\Omega=\left\{(x, z) \in \mathbb{R}^{n}, x \in \omega \text { and } 0<z<h(x)\right\} .
$$

For a function $\hat{f}$ defined in $\Omega^{\varepsilon}, f$ is defined in $\Omega$ by $f(x, z)=\hat{f}(x, \varepsilon z)$. For a function $f \in L^{p}(\Omega)$, $|f|_{p}$ still denotes the $L^{p}$-norm in $\Omega$, and similar notations hold for the other norms. Moreover, the regularizing term $\eta \Delta \boldsymbol{\sigma}^{\varepsilon \eta}$ is introduced. Denoting $\boldsymbol{\sigma}^{\varepsilon \eta}=\left(\begin{array}{cc}\sigma_{11}^{\varepsilon \eta} & \sigma_{12}^{\varepsilon \eta} \\ \sigma_{12}^{\varepsilon \eta} & \sigma_{22}^{\varepsilon \eta}\end{array}\right)$, and similar notations for the components of $\boldsymbol{G}$, it holds in $(0, \infty) \times \Omega$ :

$$
\left\{\begin{array}{l}
\rho \delta_{t} u_{1}^{\varepsilon \eta}-(1-r) \nu \Delta_{\varepsilon} u_{1}^{\varepsilon \eta}+\partial_{x} p^{\varepsilon \eta}-\partial_{x} \sigma_{11}^{\varepsilon \eta}-\frac{1}{\varepsilon} \partial_{z} \sigma_{12}^{\varepsilon \eta}=0 \\
\rho \delta_{t} u_{2}^{\varepsilon \eta}-(1-r) \nu \Delta_{\varepsilon} u_{2}^{\varepsilon \eta}+\frac{1}{\varepsilon} \partial_{z} p^{\varepsilon \eta}-\partial_{x} \sigma_{12}^{\varepsilon \eta}-\frac{1}{\varepsilon} \partial_{z} \sigma_{22}^{\varepsilon \eta}=0 \\
\nabla_{\varepsilon} \cdot \boldsymbol{u}^{\varepsilon \eta}=0 \\
\lambda\left(\delta_{t} \sigma_{11}^{\varepsilon \eta}-\tilde{N}\left(\boldsymbol{u}^{\varepsilon \eta}, \sigma_{12}^{\varepsilon \eta}\right)\right)+\sigma_{11}^{\varepsilon \eta}-\eta \Delta_{\varepsilon}\left(\sigma_{11}^{\varepsilon \eta}-G_{11}\right)-2 r \nu \partial_{x} u_{1}^{\varepsilon \eta}=0 \\
\lambda\left(\delta_{t} \sigma_{12}^{\varepsilon \eta}+\frac{1}{2} \tilde{N}\left(\boldsymbol{u}^{\varepsilon \eta}, \sigma_{11}^{\varepsilon \eta}-\sigma_{22}^{\varepsilon \eta}\right)\right)+\sigma_{12}^{\varepsilon \eta}-\eta \Delta_{\varepsilon}\left(\sigma_{12}^{\varepsilon \eta}-G_{12}\right)-r \nu\left(\partial_{x} u_{2}^{\varepsilon \eta}+\frac{1}{\varepsilon} \partial_{z} u_{1}^{\varepsilon \eta}\right)=0 \\
\lambda\left(\delta_{t} \sigma_{22}^{\varepsilon \eta}+\tilde{N}\left(\boldsymbol{u}^{\varepsilon \eta}, \sigma_{12}^{\varepsilon \eta}\right)\right)+\sigma_{22}^{\varepsilon \eta}-\eta \Delta_{\varepsilon}\left(\sigma_{22}^{\varepsilon \eta}-G_{22}\right)-2 r \nu \frac{1}{\varepsilon} \partial_{z} u_{2}^{\varepsilon \eta}=0
\end{array}\right.
$$

where the convective derivative $\delta_{t}$ is given by $\delta_{t}=\partial_{t}+\boldsymbol{u}^{\varepsilon \eta} \cdot \nabla_{\varepsilon}$. The derivation operators are defined as follows: $\nabla_{\varepsilon}=\left(\partial_{x}, \frac{1}{\varepsilon} \partial_{z}\right)$ and $\Delta_{\varepsilon}=\partial_{x}^{2}+\frac{1}{\varepsilon^{2}} \partial_{z}^{2}$. The non-linear terms $\tilde{N}$ are given by $\tilde{N}(\boldsymbol{u}, f)=\left(\partial_{x} u_{2}-\frac{1}{\varepsilon} \partial_{z} u_{1}\right) f$.

\subsection{Asymptotic expansions}

It has been proposed in [4] that when $\eta, \varepsilon$ tend zero, $\left(\boldsymbol{u}^{\varepsilon \eta}, p^{\varepsilon \eta}, \boldsymbol{\sigma}^{\varepsilon \eta}\right)$ tends formally to a triplet $\left(\boldsymbol{u}^{*}, p^{*}, \boldsymbol{\sigma}^{*}\right)$ satisfying a system that will be given later in (4.1). This analysis leads to the introduction of the following asymptotic expansions:

$$
\begin{gathered}
u_{1}^{\varepsilon \eta}=u_{1}^{*}+v_{1}^{\varepsilon \eta} \text { and } u_{2}^{\varepsilon \eta}=\varepsilon u_{2}^{*}+\varepsilon v_{2}^{\varepsilon \eta} \\
p^{\varepsilon \eta}=\frac{1}{\varepsilon^{2}} p^{*}+\frac{1}{\varepsilon^{2}} q^{\varepsilon \eta} \\
\boldsymbol{\sigma}^{\varepsilon \eta}=\frac{1}{\varepsilon} \boldsymbol{\sigma}^{*}+\frac{1}{\varepsilon} \boldsymbol{\tau}^{\varepsilon \eta}
\end{gathered}
$$

with $\boldsymbol{\sigma}^{*}=\left(\begin{array}{cc}\sigma_{11}^{*} & \sigma_{12}^{*} \\ \sigma_{12}^{*} & \sigma_{22}^{*}\end{array}\right)$, and $\boldsymbol{\tau}^{\varepsilon \eta}=\left(\begin{array}{cc}\tau_{11}^{\varepsilon \eta} & \tau_{12}^{\varepsilon \eta} \\ \tau_{12}^{\varepsilon \eta} & \tau_{22}^{\varepsilon \eta}\end{array}\right)$. If denoting $\boldsymbol{u}^{*}=\left(u_{1}^{*}, u_{2}^{*}\right)$, and $\boldsymbol{v}^{\varepsilon \eta}=\left(v_{1}^{\varepsilon \eta}, v_{2}^{\varepsilon \eta}\right)$, (3.2) becomes $\boldsymbol{u}^{\varepsilon \eta}=\boldsymbol{u}^{*}+\boldsymbol{v}^{\varepsilon \eta}$.

The scaling orders chosen for the pressure and the different components of the velocity field and 
of the stress tensor are motivated by some mathematical and physical remarks. Classically, the pressure has to be of order $1 / \varepsilon^{2}$ if the horizontal velocity is of order 1 (see [2] for the rigorous explanation). On the other hand, the stress tensor has to be of order $1 / \varepsilon$ and the Deborah number $\lambda$ of order $\varepsilon$ in order to balance the Newtonian and non-Newtonian contribution in Oldroyd equation (see [4]). Hence; let $\lambda=\varepsilon \lambda^{*}$.

A wise choice of the function $\boldsymbol{G}$ in the regularizing term is $\boldsymbol{G}=\boldsymbol{\sigma}^{*}$. The regularity of $\boldsymbol{G}$ in $\boldsymbol{H}^{2}(\Omega)$ is proved by Theorem 4.4 (where it is proved that $\partial_{x}^{2} \boldsymbol{\sigma}^{*} \in \mathcal{C}^{0}(\bar{\Omega}), \partial_{x} \partial_{z} \boldsymbol{\sigma}^{*} \in \mathcal{C}^{0}(\bar{\Omega})$ and $\partial_{z}^{2} \boldsymbol{\sigma}^{*} \in \mathcal{C}^{1}(\bar{\Omega})$, thus $\Delta \boldsymbol{\sigma}^{*} \in \boldsymbol{L}^{2}(\Omega)$ ). A formal substitution of (3.2), (3.3), (3.4) in (3.1) leads to the following system:

$$
\left\{\begin{array}{l}
\rho \mathrm{d}_{t} v_{1}^{\varepsilon \eta}-(1-r) \nu \Delta_{\varepsilon} v_{1}^{\varepsilon \eta}+\frac{1}{\varepsilon^{2}} \partial_{x} q^{\varepsilon \eta}-\frac{1}{\varepsilon} \partial_{x} \tau_{11}^{\varepsilon \eta}-\frac{1}{\varepsilon^{2}} \partial_{z} \tau_{12}^{\varepsilon \eta}=\tilde{L}_{1}^{\varepsilon \eta}+\frac{1}{\varepsilon} C_{1}+\frac{1}{\varepsilon^{2}} C_{1}^{\prime}, \\
\rho \mathrm{d}_{t} v_{2}^{\varepsilon \eta}-(1-r) \nu \Delta_{\varepsilon} v_{2}^{\varepsilon \eta}+\frac{1}{\varepsilon^{4}} \partial_{z} q^{\varepsilon \eta}-\frac{1}{\varepsilon^{2}} \partial_{x} \tau_{12}^{\varepsilon \eta}-\frac{1}{\varepsilon^{3}} \partial_{z} \tau_{22}^{\varepsilon \eta}=\frac{1}{\varepsilon^{2}} \tilde{L}_{2}^{\varepsilon \eta}+\frac{1}{\varepsilon^{3}} C_{2}+\frac{1}{\varepsilon^{4}} C_{2}^{\prime}, \\
\nabla \cdot \boldsymbol{v}^{\varepsilon \eta}=\nabla \cdot \boldsymbol{u}^{*} \\
\lambda^{*}\left(\mathrm{~d}_{t} \tau_{11}^{\varepsilon \eta}-N\left(\boldsymbol{v}^{\varepsilon \eta}, \tau_{12}^{\varepsilon \eta}\right)\right)+\frac{1}{\varepsilon} \tau_{11}^{\varepsilon \eta}-\eta \Delta_{\varepsilon} \tau_{11}^{\varepsilon \eta}-2 r \nu \partial_{x} v_{1}^{\varepsilon \eta}=\tilde{L}_{11}^{\varepsilon \eta}+\frac{1}{\varepsilon} \tilde{L}_{11}^{\varepsilon \eta}, \\
\lambda^{*}\left(\mathrm{~d}_{t} \tau_{12}^{\varepsilon \eta}+\frac{1}{2} N\left(\boldsymbol{v}^{\varepsilon \eta}, \tau_{11}^{\varepsilon \eta}-\tau_{22}^{\varepsilon \eta}\right)\right)+\frac{1}{\varepsilon} \tau_{12}^{\varepsilon \eta}-\eta \Delta_{\varepsilon} \tau_{12}^{\varepsilon \eta}-r \nu\left(\partial_{x} v_{2}^{\varepsilon \eta}+\frac{1}{\varepsilon} \partial_{z} v_{1}^{\varepsilon \eta}\right)=\tilde{L}_{12}^{\varepsilon \eta}+\frac{1}{\varepsilon} \tilde{L}_{12}^{\varepsilon \eta}, \\
\lambda^{*}\left(\mathrm{~d}_{t} \tau_{22}^{\varepsilon \eta}+N\left(\boldsymbol{v}^{\varepsilon \eta}, \tau_{12}^{\varepsilon \eta}\right)\right)+\frac{1}{\varepsilon} \tau_{22}^{\varepsilon \eta}-\eta \Delta_{\varepsilon} \tau_{22}^{\varepsilon \eta}-\frac{2 r \nu}{\varepsilon} \partial_{z} v_{2}^{\varepsilon \eta}=\tilde{L}_{22}^{\varepsilon \eta}+\frac{1}{\varepsilon} \tilde{L}_{22}^{\varepsilon \eta},
\end{array}\right.
$$

with the following notations: $\mathrm{d}_{t}=\partial_{t}+\boldsymbol{v}^{\varepsilon \eta} \cdot \nabla$ is the so-called convective derivative, the non-linear terms $N\left(\boldsymbol{v}^{\varepsilon \eta}, f\right)=\left(\varepsilon \partial_{x} v_{2}^{\varepsilon \eta}-\frac{1}{\varepsilon} \partial_{z} v_{1}^{\varepsilon \eta}\right) f$ for $f \in L^{2}(\Omega)$ and the following linear (with respect to $\left.\boldsymbol{v}^{\varepsilon \eta}\right)$ and constant terms

$$
\begin{aligned}
\tilde{L}_{1}^{\varepsilon \eta} & =\underbrace{-\rho \boldsymbol{v}^{\varepsilon \eta} \cdot \nabla u_{1}^{*}-\rho \boldsymbol{u}^{*} \cdot \nabla v_{1}^{\varepsilon \eta}}_{\mathcal{L}_{1}^{\varepsilon \eta}}-\rho \partial_{t} u_{1}^{*}-\rho \boldsymbol{u}^{*} \cdot \nabla u_{1}^{*}+(1-r) \nu \partial_{x}^{2} u_{1}^{*}, \\
C_{1} & =\partial_{x} \sigma_{11}^{*}, \\
C_{1}^{\prime} & =(1-r) \nu \partial_{z}^{2} u_{1}^{*}-\partial_{x} p^{*}+\partial_{z} \sigma_{12}^{*} ; \\
\tilde{L}_{2}^{\varepsilon \eta} & =\underbrace{-\rho \varepsilon^{2} \boldsymbol{v}^{\varepsilon \eta} \cdot \nabla u_{2}^{*}-\rho \varepsilon^{2} \boldsymbol{u}^{*} \cdot \nabla v_{2}^{\varepsilon \eta}}_{\mathcal{L}_{2}^{\varepsilon \eta}} \\
& -\rho \varepsilon^{2} \partial_{t} u_{2}^{*}-\rho \varepsilon^{2} \boldsymbol{u}^{*} \cdot \nabla u_{2}^{*}+\varepsilon^{2}(1-r) \nu \partial_{x}^{2} u_{2}^{*}+(1-r) \nu \partial_{z}^{2} u_{2}^{*}+\partial_{x} \sigma_{12}^{*}, \\
C_{2} & =\partial_{z} \sigma_{22}^{*}, \\
C_{2}^{\prime} & =\partial_{z} p^{*} .
\end{aligned}
$$

For the Oldroyd equation, the following linear (with respect to $\boldsymbol{v}$ and $\boldsymbol{\tau}$ ) and constant terms appear:

$$
\begin{aligned}
& \tilde{L}_{11}^{\varepsilon \eta}= \mathcal{L}_{11}^{\varepsilon \eta}+\lambda^{*}\left(-\partial_{t} \sigma_{11}^{*}-\boldsymbol{u}^{*} \cdot \nabla \sigma_{11}^{*}+\varepsilon \partial_{x} u_{2}^{*} \sigma_{12}^{*}\right)+2 r \nu \partial_{x} u_{1}^{*}, \\
& \text { with } \quad \mathcal{L}_{11}^{\varepsilon \eta}=\lambda^{*}\left(\varepsilon \partial_{x} u_{2}^{*} \tau_{12}^{\varepsilon \eta}+\varepsilon \partial_{x} v_{2}^{\varepsilon \eta} \sigma_{12}^{*}-\boldsymbol{v}^{\varepsilon \eta} \cdot \nabla \sigma_{11}^{*}-\boldsymbol{u}^{*} \cdot \nabla \tau_{11}^{\varepsilon \eta}\right),
\end{aligned}
$$




$$
\begin{aligned}
\tilde{L}_{11}^{\prime \varepsilon \eta} & =\underbrace{-\lambda^{*}\left(\partial_{z} u_{1}^{*} \tau_{12}^{\varepsilon \eta}+\partial_{z} v_{1}^{\varepsilon \eta} \sigma_{12}^{*}\right)}_{\mathcal{L}_{11}^{\prime \varepsilon \eta}}-\lambda^{*} \partial_{z} u_{1}^{*} \sigma_{12}^{*}-\sigma_{11}^{*} ; \\
\tilde{L}_{22}^{\varepsilon \eta}= & \mathcal{L}_{22}^{\varepsilon \eta}-\lambda^{*}\left(\partial_{t} \sigma_{22}^{*}+\boldsymbol{u}^{*} \cdot \nabla \sigma_{22}^{*}+\varepsilon \partial_{x} u_{2}^{*} \sigma_{12}^{*}\right)+2 r \nu \partial_{z} u_{2}^{*}, \\
\text { with } & \mathcal{L}_{22}^{\varepsilon \eta}=-\lambda^{*}\left(\varepsilon \partial_{x} u_{2}^{*} \tau_{12}^{\varepsilon \eta}+\varepsilon \partial_{x} v_{2} \sigma_{12}^{*}+\boldsymbol{v}^{\varepsilon \eta} \cdot \nabla \sigma_{22}^{*}+\boldsymbol{u}^{*} \cdot \nabla \tau_{22}^{\varepsilon \eta}\right), \\
\tilde{L}_{22}^{\prime \varepsilon \eta}= & \underbrace{\lambda^{*}\left(\partial_{z} u_{1}^{*} \tau_{12}^{\varepsilon \eta}+\partial_{z} v_{1}^{\varepsilon \eta} \sigma_{12}^{*}\right)}_{\mathcal{L}_{22}^{\prime \varepsilon \eta}}+\lambda^{*} \partial_{z} u_{1}^{*} \sigma_{12}^{*}-\sigma_{22}^{*} \\
\tilde{L}_{12}^{\varepsilon \eta} & =\underbrace{\left.-\frac{\lambda^{*}}{2}\left(2 \partial_{t} \sigma_{12}^{*}+2 \partial_{x} u_{2}^{*}\left(\tau_{11}^{\varepsilon \eta}-\tau_{22}^{\varepsilon \eta}\right)+\varepsilon \partial_{x} v_{2}^{\varepsilon \eta}\left(\sigma_{11}^{*}-\sigma_{22}^{*}\right)+2 \sigma_{12}^{*}+\partial_{x} u_{2}^{* \eta}\left(\sigma_{11}^{*}-\sigma_{22}^{*}\right)\right)+r \nu \sigma_{12}^{*}+2 \boldsymbol{u}^{*} \cdot \nabla \tau_{12}^{\varepsilon \eta}\right)}_{\mathcal{L}_{12}^{\varepsilon \eta}} \\
\tilde{L}_{12}^{\prime \varepsilon \eta} & =\underbrace{-\frac{\lambda^{*}}{2}\left(\partial_{z} u_{1}^{*}\left(\tau_{11}^{\varepsilon \eta}-\tau_{22}^{\varepsilon \eta}\right)+\partial_{z} v_{1}^{\varepsilon \eta}\left(\sigma_{11}^{*}-\sigma_{22}^{*}\right)\right)}_{\mathcal{L}_{12}^{\prime \varepsilon \eta} u_{2}^{*},}+\frac{\lambda^{*}}{2} \partial_{z} u_{1}^{*}\left(\sigma_{11}^{*}-\sigma_{22}^{*}\right)-\sigma_{12}^{*}+r \nu \partial_{z} u_{1}^{*} ;
\end{aligned}
$$

Note that the first order derivatives of $\boldsymbol{\sigma}^{*}$ occur in the terms $\tilde{L}^{\varepsilon \eta}$ and $C^{\varepsilon \eta}$. It will be shown in Theorem 4.4 that $\boldsymbol{\sigma}^{*}$ has sufficient regularity.

Let us observe also that equations (3.5) are similar to (3.1), except for the linear terms on the right. Thus the energy estimates will be obtained similarly for both systems, multiplying NavierStokes equation by the velocity and Oldroyd equation by the stress tensor, and integrating over $\Omega$.

\section{Limit equations}

\subsection{Limit system}

In an heuristic way, the following system of equations satisfied by $\boldsymbol{u}^{*}, p^{*}, \boldsymbol{\sigma}^{*}$ is infered from (3.5): $\boldsymbol{u}^{*}, p^{*}, \boldsymbol{\sigma}^{*}$ are steady functions solutions of:

$$
\left\{\begin{array}{l}
(1-r) \nu \partial_{z}^{2} u_{1}^{*}-\partial_{x} p^{*}+\partial_{z} \sigma_{12}^{*}=0 \\
\partial_{z} p^{*}=0 \\
\nabla \cdot \boldsymbol{u}^{*}=0 \\
\lambda^{*} \partial_{z} u_{1}^{*} \sigma_{12}^{*}+\sigma_{11}^{*}=0 \\
-\frac{\lambda^{*}}{2} \partial_{z} u_{1}^{*}\left(\sigma_{11}^{*}-\sigma_{22}^{*}\right)+\sigma_{12}^{*}=r \nu \partial_{z} u_{1}^{*}, \\
-\lambda^{*} \partial_{z} u_{1}^{*} \sigma_{12}^{*}+\sigma_{22}^{*}=0 .
\end{array}\right.
$$


This system is equipped with the following boundary condition (Dirichlet condition on the upper and lower part of the boundary, flux imposed on the lateral part of the boundary):

$$
\begin{cases}\boldsymbol{u}^{*}=0, & \text { for } z=h(x), \\ \boldsymbol{u}^{*}=(s, 0), & \text { for } z=0, \\ \int_{0}^{h(x)} \boldsymbol{u}^{*} \mathrm{~d} z \cdot n=\Phi_{0} & \text { on } \Gamma_{L} .\end{cases}
$$

The compatibility condition reads $\int \Phi_{0}=0$. Moreover, since $p^{*}$ is defined up to a constant, the mean pressure is taken to be zero: $\int_{\Omega}^{\partial \omega} p^{*}=0$.

Remark 4.1. Each equation of the preceding system (4.1) is obtained by cancelling the constant part (i.e. the part independent of $\boldsymbol{v}^{\varepsilon \eta}, q^{\varepsilon \eta}, \tau^{\varepsilon \eta}$ ) of respectively $C_{1}^{\prime}, C_{2}^{\prime}, \nabla \cdot \boldsymbol{u}^{*}, \tilde{L}_{11}^{\prime \varepsilon \eta}, \tilde{L}_{12}^{\prime \varepsilon \eta}, \tilde{L}_{22}^{\prime \varepsilon \eta}$.

\subsection{Determination of the boundary conditions}

Remark 4.2. The lateral boundary conditions on $\boldsymbol{u}^{*}$ do not depend on the ones on $\boldsymbol{u}^{\varepsilon \eta}$, but only on the flux. Therefore, different boundary conditions on $\boldsymbol{u}^{\varepsilon \eta}$ corresponding to the same flux lead to the same limit problem. This is a classical fact when passing from a two-dimensional problem to a one-dimensional problem (or similarly from a three-dimensional problem to a two-dimensional one), and has already been observed in [2] for example. Here, in order to avoid boundary layers, $\boldsymbol{u}^{\varepsilon \eta}=\boldsymbol{u}^{*}$ is imposed on the lateral part of the boundary.

Similarly, any value of $\boldsymbol{\sigma}^{\varepsilon \eta}$ on the boundary leads to the same limit problem. Again, in order to avoid boundary layers, well-prepared boundary conditions are also chosen for $\boldsymbol{\sigma}^{\varepsilon \eta}$.

The preceding remark allows to define precisely the function $\boldsymbol{J}^{\varepsilon}$ introduced in (2.3). Since $\left.\boldsymbol{u}^{*}\right|_{\Gamma_{L}} \in \boldsymbol{H}^{1 / 2}\left(\Gamma_{L}\right)$, it is possible to construct $\boldsymbol{J}^{\varepsilon} \in \boldsymbol{H}^{1 / 2}(\partial \Omega)$ satisfying $\left.\boldsymbol{J}^{\varepsilon}\right|_{z=h}=0,\left.\boldsymbol{J}^{\varepsilon}\right|_{z=0}=(s, 0)$ and $\left.\boldsymbol{J}^{\varepsilon}\right|_{\Gamma_{L}}=\left.\boldsymbol{u}^{*}\right|_{\Gamma_{L}}$. Therefore, the boundary conditions on $\boldsymbol{u}^{\varepsilon \eta}$ become

$$
\begin{cases}\boldsymbol{u}^{\varepsilon \eta}=0, & \text { for } z=h(x), \\ \boldsymbol{u}^{\varepsilon \eta}=(s, 0), & \text { for } z=0, \\ \boldsymbol{u}^{\varepsilon \eta}=\boldsymbol{u}^{*} & \text { on } \Gamma_{L} .\end{cases}
$$

Thus $\left.\boldsymbol{u}^{\varepsilon \eta}\right|_{\partial \Omega}=\left.\boldsymbol{u}^{*}\right|_{\partial \Omega}$, and $\boldsymbol{v}^{\varepsilon \eta}$ will satisfy zero boundary conditions: $\left.\boldsymbol{v}^{\varepsilon \eta}\right|_{\partial \Omega}=0$.

Moreover, since $\boldsymbol{\sigma}^{*} \in \boldsymbol{H}^{1}(\Omega)$ (see Theorem 4.4 for this regularity result), $\boldsymbol{\theta}^{\varepsilon}$ can be defined as follows:

$$
\boldsymbol{\theta}^{\varepsilon}=\left.\boldsymbol{\sigma}^{*}\right|_{\Gamma_{+}} \in \boldsymbol{H}^{1 / 2}\left(\Gamma_{+}\right) .
$$

Therefore

$$
\left.\boldsymbol{\sigma}^{\varepsilon \eta}\right|_{\Gamma_{+}}=\left.\boldsymbol{\sigma}^{*}\right|_{\Gamma_{+}},
$$

and this implies that $\left.\boldsymbol{\tau}^{\varepsilon \eta}\right|_{\Gamma_{+}}=0$. 
On the other part $\Gamma_{-}$of the boundary, $\boldsymbol{\sigma}^{\varepsilon \eta}$ is chosen such that $\left.\boldsymbol{\sigma}^{\varepsilon \eta} \cdot n\right|_{\Gamma_{-}}=\left.\boldsymbol{\sigma}^{*} \cdot n\right|_{\Gamma_{-}}$, for example $\left.\boldsymbol{\sigma}^{\varepsilon \eta}\right|_{\Gamma_{-}}=\left.\boldsymbol{\sigma}^{*}\right|_{\Gamma_{-}}$

\subsection{Existence of a solution to the limit problem}

System (4.1)-(4.2) has already been studied in [4].

Theorem 4.3. Assume that $r<8 / 9$. Then system (4.1)-(4.2) has a unique solution satisfying

$$
\boldsymbol{u}^{*} \in \boldsymbol{L}^{2}(\Omega), \quad \partial_{z} \boldsymbol{u}^{*} \in \boldsymbol{L}^{2}(\Omega), \quad p^{*} \in H^{1}(\omega), \quad \boldsymbol{\sigma}^{*} \in \boldsymbol{L}^{2}(\Omega) .
$$

Proof. This result has been proved in [4].

This existence result is not sufficient for this study. Therefore, the following stronger regularity result is proved on the limit problem (4.1)-(4.2).

Theorem 4.4. Assume $r<2 / 9$. If $h \in H^{k}(\omega)$, for $k \in \mathbb{N}^{*}$, then the unique solution $\left(\boldsymbol{u}^{*}, p^{*}, \boldsymbol{\sigma}^{*}\right)$ of the system (4.1)-(4.2) satisfies

$$
\begin{gathered}
p^{*} \in \mathcal{C}^{k+1}(\bar{\omega}), \quad u_{1}^{*}, \partial_{z} u_{1}^{*}, \partial_{z}^{2} u_{1}^{*} \in \mathcal{C}^{k+1}(\bar{\Omega}), \quad \boldsymbol{\sigma}^{*}, \partial_{z} \boldsymbol{\sigma}^{*} \in \mathcal{C}^{k+1}(\bar{\Omega}), \\
\partial_{x} u_{1}^{*} \in \mathcal{C}^{k}(\bar{\Omega}), \quad u_{2}^{*}, \partial_{z} u_{2}^{*}, \partial_{z}^{2} u_{2}^{*} \in \mathcal{C}^{k}(\bar{\Omega}), \quad \partial_{x} \boldsymbol{\sigma}^{*} \in \mathcal{C}^{k}(\bar{\Omega}) \\
\partial_{x} u_{2}^{*} \in \mathcal{C}^{k-1}(\bar{\Omega}) .
\end{gathered}
$$

Proof. Let us observe that system (4.1) can be expressed as a system on $u_{1}^{*}, p^{*}$ only. Using (4.1), $\sigma_{11}^{*}, \sigma_{22}^{*}$ can be expressed as functions of $\sigma_{12}^{*}$ and $\partial_{z} u_{1}^{*}$. Indeed, from the fourth and the last equations of (4.1), it holds that

$$
\sigma_{22}^{*}=-\sigma_{11}^{*}=\lambda^{*} \partial_{z} u_{1}^{*} \sigma_{12}^{*}
$$

Moreover, the divergence-free equation can be rewritten in order to eliminate $u_{2}^{*}$. Integrating this equation between $z=0$ and $z=h$, and using the fact that $\left.u_{2}^{*}\right|_{z=0}=\left.u_{2}^{*}\right|_{z=h}=\left.u_{1}^{*}\right|_{z=h}=0$, it follows:

$$
\partial_{x}\left(\int_{0}^{h} u_{1}^{*} \mathrm{~d} z\right)=0 .
$$

Thus, the system in $u_{1}^{*}, p^{*}$ can be written in the following form:

$$
\left\{\begin{array}{l}
-\nu(1-r) \partial_{z}^{2} u_{1}^{*}-\partial_{z} \sigma_{12}^{*}+\partial_{x} p^{*}=0, \quad \text { with } \sigma_{12}^{*}=\frac{\nu r \partial_{z} u_{1}^{*}}{1+\lambda^{* 2}\left|\partial_{z} u_{1}^{*}\right|^{2}} \\
\partial_{z} p^{*}=0 \\
\partial_{x}\left(\int_{0}^{h} u_{1}^{*} \mathrm{~d} z\right)=0
\end{array}\right.
$$

equipped with the boundary conditions stated in (4.2) and the condition $\int_{\Omega} p^{*}=0$. 
For the sake of readability, the superscripts * are omitted in the rest of this section.

Denote $q=\partial_{x} p$. Let $\phi \in \mathcal{C}^{\infty}(\mathbb{R})$ defined by $\phi(t)=\nu(1-r) t+\frac{\nu r t}{1+\lambda^{2} t^{2}}$. The first equation of (4.8) becomes $q=\partial_{z}\left(\phi\left(\partial_{z} u_{1}\right)\right)$.

A simple study of function $\phi$ allows to show the following properties:

$$
0<\nu\left(1-\frac{9 r}{8}\right)<\left|\phi^{\prime}\right|_{\infty}<\nu, \quad \text { and } \quad \phi(t) \underset{t \rightarrow \pm \infty}{\longrightarrow} \pm \infty
$$

Therefore the function $\phi$ is invertible, and $\psi=\phi^{-1}$ belongs to $\mathcal{C}^{\infty}(\mathbb{R})$. Moreover, $\psi$ is an increasing function as $\phi$. Integrating $q=\partial_{z}\left(\phi\left(\partial_{z} u_{1}\right)\right)$ with respect to $z$ between 0 and $z$, the first equation of (4.8) becomes:

$$
\phi\left(\partial_{z} u_{1}(x, z)\right)=q(x) z+\kappa(x),
$$

where $\kappa(x)$ is a integration constant. Therefore, it follows that

$$
\partial_{z} u_{1}(x, z)=\psi(q(x) z+\kappa(x)) .
$$

Since $\left.u_{1}\right|_{z=0}=s$, the integration between 0 and $z$ of the preceding equation yields:

$$
u_{1}(x, z)=s+\int_{0}^{z} \psi(q(x) t+\kappa(x)) \mathrm{d} t
$$

The boundary condition $\left.u_{1}\right|_{h(x)}=0$ implies also:

$$
\int_{0}^{h(x)} \psi(q(x) t+\kappa(x))+s=0 .
$$

For $(h, q, s, \kappa) \in \mathbb{R}^{4}$, let us introduce $F(h, q, s, \kappa)=\int_{0}^{h} \psi(q t+\kappa)+s$.

Lemma 4.5. For any $(h, q, s) \in \mathbb{R}^{3}$ there exists an unique $\kappa \in \mathbb{R}$ such that $F(h, q, s, \kappa)=0$.

Proof. - If such an $\kappa$ exists, it is unique from the implicit function theorem, since for all $(h, q, s, \kappa) \in \mathbb{R}^{4}$

$$
\frac{\partial F}{\partial \kappa}(h, q, s, \kappa)=\int_{0}^{h} \psi^{\prime}(q t+\kappa) \mathrm{d} t>0 .
$$

- The following limits are computed, using the fact that $\lim _{t \rightarrow \pm \infty} \psi(t)= \pm \infty$ :

$$
\lim _{\kappa \rightarrow+\infty} F(h, q, s, \kappa)=+\infty \quad \text { and } \quad \lim _{\kappa \rightarrow-\infty} F(h, q, s, \kappa)=-\infty
$$

Therefore, there exists $\kappa \in \mathbb{R}$ such that $F(h, q, s, \kappa)=0$. Let us denote $K(h, q, s)=\kappa$. By the implicit function theorem, $K \in \mathcal{C}^{\infty}\left(\mathbb{R}^{3}\right)$. 
Therefore, the following expression holds for $(h, q, s) \in \mathbb{R}^{3}$ :

$$
F(h, q, s, K(h, q, s))=0 .
$$

It is now possible to obtain an information on the sign of $\partial_{q} K$. Indeed, deriving the expression (4.12) with respect to $q$, it follows

$$
\partial_{q} F+\partial_{\kappa} F \partial_{q} K=0
$$

For $h>0$, since $\partial_{q} F=\int_{0}^{h} t \psi^{\prime}(q t+\kappa) \mathrm{d} t>0$ and $\partial_{a} F=\int_{0}^{h} \psi^{\prime}(q t+\kappa) \mathrm{d} t>0, \partial_{q} K$ is strictly negative.

Now, using equation (4.7) and the expression (4.10) for $u$, it follows:

$$
\int_{0}^{h(x)} \int_{0}^{z} \partial_{x}(\psi(q(x) t+K(h(x), q(x), s))) \mathrm{d} t \mathrm{~d} z=0 .
$$

or if changing the direction of integration

$$
\int_{0}^{h(x)}(h(x)-t) \partial_{x}(\psi(q(x) t+K(h(x), q(x), s))) \mathrm{d} t=0
$$

This can be rewritten as

$$
\begin{aligned}
q^{\prime}(x) & \int_{0}^{h(x)}(h(x)-t)\left(\left(t+\partial_{q} K(h(x), q(x), s)\right) \psi^{\prime}(q(x) t+K(h(x), q(x), s)) \mathrm{d} t\right. \\
& =-\int_{0}^{h(x)}(h(x)-t)\left(h^{\prime}(x) \partial_{h} K(h(x), q(x), s)\right) \psi^{\prime}(q(x) t+K(h(x), q(x), s)) \mathrm{d} t
\end{aligned}
$$

which can be seen as an ordinary differential equation in $q$. Let

$$
\begin{aligned}
& U(x, q)=\int_{0}^{h(x)}(h(x)-t)\left(t+\partial_{q} K(h(x), q, s)\right) \psi^{\prime}(q t+K(h(x), q, s)) \mathrm{d} t \\
& V(x, q)=\int_{0}^{h(x)}(h(x)-t)\left(h^{\prime}(x) \partial_{h} K(h(x), q, s)\right) \psi^{\prime}(q t+K(h(x), q, s)) \mathrm{d} t .
\end{aligned}
$$

The differential equation becomes $U(x, q(x)) q^{\prime}(x)=-V(x, q(x))$ for $x \in \omega$. Note that this equation is in some sense a generalized Reynolds equation for the pressure.

Lemma 4.6. Let $r<2 / 9$. Then $U(x, q)<0$ for any $(x, q) \in \omega \times \mathbb{R}$.

Proof. Let $(x, q) \in \omega \times \mathbb{R}$. Equation (4.11) and the definition (4.12) of $K$ imply:

$$
\int_{0}^{h(x)} \psi(q t+K(h(x), q, s)) \mathrm{d} t=-s,
$$


which becomes, after derivation with respect to $q$

$$
\int_{0}^{h(x)}\left(t+\partial_{q} K(h(x), q, s)\right) \psi^{\prime}(q t+K(h(x), q, s)) \mathrm{d} t=0 .
$$

With the notation $K^{\prime}(x, q)=\partial_{q} K(h(x), q, s),(4.13)$ implies

$$
K^{\prime}(x, q)=-\frac{\int_{0}^{h(x)} t \psi^{\prime}(q t+K(h(x), q, s)) \mathrm{d} t}{\int_{0}^{h(x)} \psi^{\prime}(q t+K(h(x), q, s)) \mathrm{d} t} .
$$

Now, using this expression, $U(x, q)$ can be simplified:

$$
U(x, q)=\int_{0}^{h(x)}-t\left(t+\partial_{q} K(h(x), q, s)\right) \psi^{\prime}(q t+K(h(x), q, s)) \mathrm{d} t .
$$

Recalling the estimate of $|\phi|_{\infty}$ in (4.9), it follows that for any $t \in \mathbb{R}$ :

$$
\frac{1}{\nu}<\psi^{\prime}(t)=\frac{1}{\phi^{\prime}(\psi(t)}<\frac{1}{\nu(1-9 r / 8)}
$$

Let $m=\frac{1}{\nu}, M=\frac{1}{\nu(1-9 r / 8)}$. Then

$$
-\frac{b h(x)}{2 m} \leq K^{\prime}(x, q) \leq-\frac{a h(x)}{2 M} .
$$

Now, (4.14) implies that:

$$
\begin{aligned}
h(x)^{3}\left(\frac{m}{3}-\frac{M}{4}\right) & =\int_{0}^{h(x)} t m\left(t-\frac{M h(x)}{2 m}\right) \\
& \leq-U(x, q) \leq \int_{0}^{h(x)} t M\left(t-\frac{m h(x)}{2 M}\right)=h(x)^{3}\left(\frac{M}{3}-\frac{m}{4}\right) .
\end{aligned}
$$

In order to prove that $U$ remains strictly negative, it suffices to prove that $0<\frac{m}{3}-\frac{M}{4}$, i.e. that $\frac{m}{M}>\frac{3}{4}$, which is satisfied under the condition $r<\frac{2}{9}$.

It is possible to apply Picard-Lindelöf theorem (or Cauchy-Lipschitz theorem) to the ordinary differential equation $-U(x, q(x)) q^{\prime}(x)=V(x, q(x))$, as $U$ remains strictly negative by Lemma 4.6. Since $\psi$ and $K$ are $\mathcal{C}^{\infty}$-functions, the regularity of $q^{\prime}$ is determined by the regularity of $q$ and $h$. By hypothesis, $h$ belongs to $H^{k}(\omega)$, with $k \in \mathbb{N}$, hence $h \in L^{2}(\omega)$. Moreover, Theorem 4.3 implies that $q \in L^{2}(\omega)$. Thus $q^{\prime} \in L^{2}(\omega)$, which means $q \in H^{1}(\omega)$.

Iterating this process as long as $h$ is regular, $h \in H^{k}(\omega)$ and $q \in H^{k}(\omega)$ implies that $q^{\prime} \in H^{k}(\omega)$, thus $\partial_{x} p=q \in H^{k+1}(\omega)$, and $p \in H^{k+2}(\omega)$. By the classical Sobolev embedding, $p$ belongs to $\mathcal{C}^{k+1}(\bar{\omega})$. 
Last, recalling the expression (4.10), it follows that $u_{1} \in \mathcal{C}^{k+1}(\bar{\omega})$, and, taking the first and second derivatives of (4.10) with respect to $z$, that $\partial_{z} u_{1}, \partial_{z}^{2} u_{1}$ also belong to $\mathcal{C}^{k+1}(\bar{\omega})$.

As observed in the introduction of the proof, $\boldsymbol{\sigma}$ and $u_{2}$ are given as functions of $p, u_{1}$, and the needed regularity follows.

Remark 4.7. Since in practical applications, $h$ is very regular $\left(h \in C^{\infty}(\bar{\omega})\right)$, the preceding theorem gives as much regularity as wanted. In particular, the following result will be useful subsequently.

Corollary 4.8. Assume $r<2 / 9$. If $h \in H^{1}(\omega)$, then the unique solution $\left(\boldsymbol{u}^{*}, p^{*}, \boldsymbol{\sigma}^{*}\right)$ of the system (4.1)-(4.2) satisfies

$$
\begin{gathered}
p^{*} \in \mathcal{C}^{2}(\bar{\omega}), \quad u_{1}^{*}, \partial_{z} u_{1}^{*}, \partial_{z}^{2} u_{1}^{*} \in \mathcal{C}^{2}(\bar{\Omega}), \quad \boldsymbol{\sigma}^{*}, \partial_{z} \boldsymbol{\sigma}^{*} \in \mathcal{C}^{2}(\bar{\Omega}), \\
\partial_{x} u_{1}^{*} \in \mathcal{C}^{1}(\bar{\Omega}), \quad u_{2}^{*}, \partial_{z} u_{2}^{*}, \partial_{z}^{2} u_{2}^{*} \in \mathcal{C}^{1}(\bar{\Omega}), \quad \partial_{x} \boldsymbol{\sigma}^{*} \in \mathcal{C}^{1}(\bar{\Omega}), \\
\partial_{x} u_{2}^{*} \in \mathcal{C}^{0}(\bar{\Omega}) .
\end{gathered}
$$

Proof. It suffices to take $k=1$ in the preceding theorem 4.4.

\section{Convergence of the remainders}

\subsection{Equations on the remainders}

From now on, the superscript ${ }^{\varepsilon \eta}$ are dropped although the functions still depend on $\varepsilon$ and $\eta$. Using the equations (4.1), system (3.5) becomes

$$
\left\{\begin{array}{l}
\rho \mathrm{d}_{t} v_{1}-(1-r) \nu \Delta_{\varepsilon} v_{1}+\frac{1}{\varepsilon^{2}} \partial_{x} q-\frac{1}{\varepsilon} \partial_{x} \tau_{11}-\frac{1}{\varepsilon^{2}} \partial_{z} \tau_{12}=L_{1}+\frac{1}{\varepsilon} C_{1} \\
\rho \mathrm{d}_{t} v_{2}-(1-r) \nu \Delta_{\varepsilon} v_{2}+\frac{1}{\varepsilon^{4}} \partial_{x} q-\frac{1}{\varepsilon^{2}} \partial_{x} \tau_{12}-\frac{1}{\varepsilon^{3}} \partial_{z} \tau_{22}=\frac{1}{\varepsilon^{2}} L_{2}+\frac{1}{\varepsilon^{3}} C_{2} \\
\nabla \cdot \boldsymbol{v}=0 \\
\lambda^{*} \mathrm{~d}_{t} \tau_{11}-\lambda^{*} N\left(\boldsymbol{v}, \tau_{12}\right)+\frac{1}{\varepsilon} \tau_{11}-\eta \Delta_{\varepsilon} \tau_{11}-2 r \nu \partial_{x} v_{1}=L_{11}+\frac{1}{\varepsilon} L_{11}^{\prime}+\eta \Delta_{\varepsilon} \sigma_{11}^{*} \\
\lambda^{*} \mathrm{~d}_{t} \tau_{12}+\frac{\lambda^{*}}{2} N\left(\boldsymbol{v}, \tau_{11}-\tau_{22}\right)+\frac{1}{\varepsilon} \tau_{12}-\eta \Delta_{\varepsilon} \tau_{12}-r \nu\left(\partial_{x} v_{2}+\frac{1}{\varepsilon} \partial_{z} v_{1}\right)=L_{12}+\frac{1}{\varepsilon} L_{12}^{\prime}+\eta \Delta_{\varepsilon} \sigma_{12}^{*} \\
\lambda^{*} \mathrm{~d}_{t} \tau_{22}+\lambda^{*} N\left(\boldsymbol{v}, \tau_{12}\right)+\frac{1}{\varepsilon} \tau_{22}-\eta \Delta_{\varepsilon} \tau_{22}-\frac{2 r \nu}{\varepsilon} \partial_{z} v_{2}=L_{22}+\frac{1}{\varepsilon} L_{22}^{\prime}+\eta \Delta_{\varepsilon} \sigma_{22}^{*}
\end{array}\right.
$$

with the new quantities

$$
\begin{aligned}
L_{1} & =\mathcal{L}_{1}-\rho \boldsymbol{u}^{*} \cdot \nabla u_{1}^{*}+(1-r) \nu \partial_{x}^{2} u_{1}^{*}, \\
L_{2} & =\mathcal{L}_{2}-\rho \varepsilon^{2} \boldsymbol{u}^{*} \cdot \nabla u_{2}^{*}+(1-r) \nu \partial_{x}^{2} u_{2}^{*}+(1-r) \nu \partial_{z} u_{2}^{*}+\partial_{x} \sigma_{12}^{*}, \\
L_{11} & =\mathcal{L}_{11}+\lambda^{*}\left(-\boldsymbol{u}^{*} \cdot \nabla \sigma_{11}^{*}+\varepsilon \partial_{x} u_{2}^{*} \sigma_{12}^{*}\right)+2 r \nu \partial_{x} u_{1}^{*}, \\
L_{11}^{\prime} & =\mathcal{L}_{11}^{\prime}, \\
L_{12} & =\mathcal{L}_{12}-\frac{\lambda^{*}}{2}\left(2 \boldsymbol{u}^{*} \cdot \nabla \sigma_{12}^{*}+\partial_{x} u_{2}^{*}\left(\sigma_{11}^{*}-\sigma_{22}^{*}\right)\right)+r \nu \varepsilon \partial_{x} u_{2}^{*}, \\
L_{12}^{\prime} & =\mathcal{L}_{12}^{\prime},
\end{aligned}
$$




$$
\begin{aligned}
& L_{22}=\mathcal{L}_{22}-\lambda^{*}\left(\boldsymbol{u}^{*} \cdot \nabla \sigma_{22}^{*}+\varepsilon \partial_{x} u_{2}^{*} \sigma_{12}^{*}\right)+2 r \nu \partial_{z} u_{2}^{*}, \\
& L_{22}^{\prime}=\mathcal{L}_{22}^{\prime}
\end{aligned}
$$

and with the initial and boundary conditions

$$
\left.\boldsymbol{v}\right|_{t=0}=\boldsymbol{u}_{0}-\boldsymbol{u}^{*},\left.\quad \boldsymbol{\tau}\right|_{t=0}=\boldsymbol{\sigma}_{0}-\boldsymbol{\sigma}^{*},\left.\quad \boldsymbol{v}\right|_{\partial \Omega}=0,\left.\quad \boldsymbol{\tau}\right|_{\Gamma_{+}}=0
$$

Let us observe that both initial conditions $\left.\boldsymbol{v}\right|_{t=0}$ and $\left.\boldsymbol{\tau}\right|_{t=0}$ belong to $\boldsymbol{L}^{2}(\Omega) . \boldsymbol{v}, q$ and $\boldsymbol{\tau}$ are defined by (3.2), (3.3), (3.4). From the existence theorem 2.1 for $(\boldsymbol{u}, p, \boldsymbol{\sigma})$ and theorem 4.3 for $\left(\boldsymbol{u}^{*}, p^{*}, \boldsymbol{\sigma}^{*}\right)$, it follows that system (5.1) admits a solution $(\boldsymbol{v}, q, \boldsymbol{\tau}) \in L^{2}\left(0, \infty, \boldsymbol{H}^{1}(\Omega)\right) \times L^{2}\left(0, \infty, L^{2}(\Omega)\right) \times$ $\mathcal{C}\left(0, \infty, \boldsymbol{L}^{2}(\Omega)\right)$ for $r<8 / 9$.

\subsection{Convergence of $v$ and $\tau$}

Before starting the a priori estimates, let us explain how the non-linear terms in (5.1) are handled. The non-linear terms $\boldsymbol{v} \cdot \nabla \boldsymbol{v}$ of Navier-Stokes equation and $\boldsymbol{v} \cdot \nabla \boldsymbol{\tau}$ of Oldroyd equation are treated with the following Lemma 5.1. On the other hand, the non-linear terms $N(\boldsymbol{v}, \boldsymbol{\tau})=$ $\left(\varepsilon \partial_{x} v_{2}-\frac{1}{\varepsilon} \partial_{z} v_{1}\right) \boldsymbol{\tau}$ in (5.1d)-(5.1f) are zero when multiplied by $\boldsymbol{\tau}$.

Lemma 5.1. Let $\boldsymbol{n}$ be the exterior normal of the domain $\Omega$. Let $\boldsymbol{\phi} \in \boldsymbol{H}^{1}(\Omega)$ be a vector field satisfying $\nabla \cdot \boldsymbol{\phi}=0$ and $\left.\boldsymbol{\phi} \cdot \boldsymbol{n}\right|_{\partial \Omega}=0$. Let $w \in H^{1}(\Omega)$. Then

$$
\int_{\Omega} \phi \cdot \nabla w w=0
$$

Proof. By integration by parts:

$$
\int_{\Omega} \phi \cdot \nabla w w=-\int_{\Omega} \underbrace{\nabla \cdot \boldsymbol{\phi}}_{=0} \cdot w^{2}-\int_{\Omega} \phi \cdot \nabla w w+\int_{\partial \Omega} \underbrace{\boldsymbol{\phi} \cdot \boldsymbol{n}}_{=0} w^{2}=0 .
$$

The classical approach consists in obtaining a priori estimates for $\boldsymbol{v}$.

Proposition 5.2. Let $(\boldsymbol{v}, q, \boldsymbol{\tau})$ be a solution of (5.1). Then $\boldsymbol{v}=\left(v_{1}, v_{2}\right)$ satisfy the following inequality for $\varepsilon$ small enough:

$$
r \nu \rho \frac{d}{d t}\left(\left|v_{1}\right|^{2}+\left|\varepsilon v_{2}\right|^{2}\right)+\frac{3}{2} r(1-r) \nu^{2}\left(\left|\nabla_{\varepsilon} v_{1}\right|^{2}+\left|\varepsilon \nabla_{\varepsilon} v_{2}\right|^{2}\right) \leq-\mathcal{D}_{1}-\mathcal{D}_{2}+C,
$$

where $\mathcal{D}_{1}=\frac{2 r \nu}{\varepsilon} \int_{\Omega} \tau_{11} \partial_{x} v_{1}+\frac{2 r \nu}{\varepsilon^{2}} \int_{\Omega} \tau_{12} \partial_{z} v_{1}, \mathcal{D}_{2}=2 r \nu \int_{\Omega} \tau_{12} \partial_{x} v_{2}+\frac{2 r \nu}{\varepsilon} \int_{\Omega} \tau_{22} \partial_{z} v_{2}$ and $C$ is a constant independent of $\varepsilon$.

Proof. The proof consists in obtaining classical a priori estimates on both $v_{1}$ and $v_{2}$. 
Step 1. Let us multiply (5.1a) by $v_{1}$ and integrate over $\Omega$. Observe that $v_{1}$ is regular enough to do so. Since $\left.\boldsymbol{v}\right|_{\partial \Omega}=0$, the boundary terms in the integration by parts are all zero. For example $-\int_{\Omega} \Delta_{\varepsilon} v_{1} v_{1}=\int_{\Omega}\left|\nabla_{\varepsilon} v_{1}\right|^{2}$. Moreover, the convection terms $\int_{\Omega} \boldsymbol{v} \cdot \nabla v_{1} v_{1}$ contained in $\int_{\Omega} \mathrm{d}_{t} v_{1} v_{1}$ are equal to zero by Lemma 5.1 , since $\nabla \cdot \boldsymbol{v}=0$ and $\left.\boldsymbol{v}\right|_{\partial \Omega}=0$. It follows:

$$
\frac{\rho}{2} \frac{\mathrm{d}}{\mathrm{d} t}\left|v_{1}\right|^{2}+(1-r) \nu\left|\nabla_{\varepsilon} v_{1}\right|^{2}-\frac{1}{\varepsilon^{2}} \int_{\Omega} q \partial_{x} v_{1}=\underbrace{-\frac{1}{\varepsilon} \int_{\Omega} \tau_{11} \partial_{x} v_{1}-\frac{1}{\varepsilon^{2}} \int_{\Omega} \tau_{12} \partial_{z} v_{1}}_{-\mathcal{D}_{1} / 2 r \nu}+\int_{\Omega} L_{1} v_{1}+\frac{1}{\varepsilon} \int_{\Omega} C_{1} v_{1} .
$$

It remains to estimate the terms $\int_{\Omega} L_{1} v_{1}$ and $\int_{\Omega} C_{1} v_{1}$.

Main idea Estimates of the form: $\int_{\Omega} L_{1} v_{1}+\frac{1}{\varepsilon} \int_{\Omega} C_{1} v_{1} \leq C+\kappa_{1}\left|\nabla_{\varepsilon} v_{1}\right|^{2}+\kappa_{2}\left|\partial_{z} v_{2}\right|^{2}$ will be proved, where $C$ is a constant independent of $\varepsilon$ and where the constants $\kappa_{1}, \kappa_{2}$ satisfy $\kappa_{1}, \kappa_{2}<(1-r) \nu / 4$. These constants will be precised later in the proof.

In the following, $C, c_{i}$ and $M_{i}$ will denote some constants independent of $\varepsilon$ and $\eta$, which might depend on $|\Omega|$, on the physical parameters of the problem and on $\boldsymbol{u}^{*}, \boldsymbol{\sigma}^{*}$ in sufficiently regular norms.

- Let us estimate first the linear (with respect to $\boldsymbol{v}$ ) term $\mathcal{L}_{1}$ of $L_{1}$. To this end, Poincaré inequality is useful: for $f \in L^{2}(\Omega)$, with $\left.f\right|_{z=h}=0,|f| \leq C_{P}\left|\partial_{z} f\right|$. The constant $C_{P}$ only depends on $\Omega$.

$\star \rho \int_{\Omega} v_{1} \partial_{x} u_{1}^{*} v_{1} \leq \rho\left|\partial_{x} u_{1}^{*}\right|_{\infty}\left|v_{1}\right|^{2} \leq \rho \varepsilon^{2} C_{P}^{2}\left|\partial_{x} u_{1}^{*}\right|_{\infty}\left|\frac{1}{\varepsilon} \partial_{z} v_{1}\right|^{2}=: M_{1} \varepsilon^{2}\left|\frac{1}{\varepsilon} \partial_{z} v_{1}\right|^{2}$.

Note that by Theorem 4.4, $\partial_{x} u_{1}^{*} \in L^{\infty}(\Omega)$. In the following, all the regularity results used in the estimates also follow from Theorem 4.4.

$\star$ For the next term, Poincaré inequality is combined with Young inequality:

$$
\begin{aligned}
\rho \int_{\Omega} v_{2} \partial_{z} u_{1}^{*} v_{1} \leq \rho\left|\partial_{z} u_{1}^{*}\right|_{\infty}\left|v_{2}\right|\left|v_{1}\right| & \leq \rho C_{P}^{2}\left|\partial_{z} u_{1}^{*}\right|_{\infty}\left|\partial_{z} v_{2}\right|\left|\partial_{z} v_{1}\right| \\
& \leq \underbrace{\rho C_{P}^{2}\left|\partial_{z} u_{1}^{*}\right|_{\infty}}_{=: M_{2}}\left(\frac{\varepsilon}{2}\left|\partial_{z} v_{2}\right|^{2}+\frac{\varepsilon}{2}\left|\frac{1}{\varepsilon} \partial_{z} v_{1}\right|^{2}\right)
\end{aligned}
$$

$\star$ In a similar way:

$$
\rho \int_{\Omega} \boldsymbol{u}^{*} \cdot \nabla v_{1} v_{1} \leq \underbrace{\rho C_{P}\left|u_{1}^{*}\right|_{\infty}}_{=: M_{3}}\left(\frac{\varepsilon}{2}\left|\partial_{x} v_{1}\right|^{2}+\frac{\varepsilon}{2}\left|\frac{1}{\varepsilon} \partial_{z} v_{1}\right|^{2}\right)+\varepsilon^{2} \underbrace{\rho C_{P}\left|u_{2}^{*}\right|_{\infty}}_{=: M_{4}}\left|\frac{1}{\varepsilon} \partial_{z} v_{1}\right|^{2} .
$$

Observe here that it was not possible to apply Lemma 5.1, since $\left.\boldsymbol{u}^{*} \cdot \boldsymbol{n}\right|_{\partial \Omega} \neq 0$.

- It remains the easier terms of $L_{1}$ and $C_{1}$ (the ones which do not depend on $\boldsymbol{v}$ ). 
* The first term is treated using again Poincaré and Young inequalities:

$\rho \int_{\Omega} \boldsymbol{u}^{*} \cdot \nabla u_{1}^{*} v_{1} \leq \rho C_{P}\left|\boldsymbol{u}^{*}\right|_{\infty}\left|\nabla u_{1}^{*}\right|\left|\partial_{z} v_{1}\right| \leq \frac{1}{2}\left(\rho C_{P}\left|\boldsymbol{u}^{*}\right|_{\infty}\left|\nabla u_{1}^{*}\right|\right)^{2}+\frac{\varepsilon^{2}}{2}\left|\frac{1}{\varepsilon} \partial_{z} v_{1}\right|^{2} \leq C+\frac{\varepsilon^{2}}{2}\left|\frac{1}{\varepsilon} \partial_{z} v_{1}\right|^{2}$.

$\star$ Similarly, $(1-r) \nu \int_{\Omega} \partial_{x}^{2} u_{1}^{*} v_{1} \leq C+\frac{\varepsilon^{2}}{2}\left|\frac{1}{\varepsilon} \partial_{z} v_{1}\right|^{2}$.

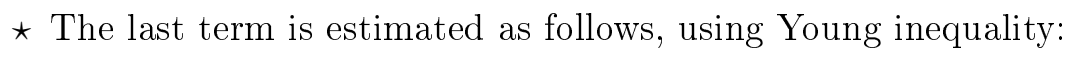

$$
\frac{1}{\varepsilon} \int_{\Omega} \partial_{x} \sigma_{11}^{*} v_{1} \leq \frac{1}{4 c}\left|\partial_{x} \sigma_{11}^{*}\right|^{2}+c\left|\frac{1}{\varepsilon} \partial_{z} v_{1}\right|^{2} \leq C+c\left|\frac{1}{\varepsilon} \partial_{z} v_{1}\right|^{2}
$$

where $c$ is a positive constant independent of $\varepsilon$ that can be chosen arbitrarily.

Now, let us choose $\varepsilon$ and $c$ small enough such that all constants satisfy:

$$
M_{1} \varepsilon^{2}, \frac{M_{2} \varepsilon}{2}, \frac{M_{3} \varepsilon}{2}, M_{4} \varepsilon^{2}, \frac{\varepsilon^{2}}{2}, c \leq \frac{(1-r) \nu}{36} .
$$

Step 2. Let us multiply (5.1b) by $\varepsilon^{2} v_{2}$ and integrate over $\Omega$. Again, the boundary terms in the integrations by parts vanish, since $\left.v_{2}\right|_{\partial \Omega}=0$, and the convection terms are equal to zero since $\nabla \cdot \boldsymbol{v}=0$ and $\left.\boldsymbol{v}\right|_{\partial \Omega}=0$ (by Lemma 5.1). It follows:

$$
\frac{\rho \varepsilon^{2}}{2} \frac{\mathrm{d}}{\mathrm{d} t}\left|v_{2}\right|^{2}+(1-r) \nu\left|\varepsilon \nabla_{\varepsilon} v_{2}\right|^{2}-\frac{1}{\varepsilon^{2}} \int_{\Omega} q \partial_{z} v_{2}=\underbrace{-\int_{\Omega} \tau_{12} \partial_{x} v_{2}-\frac{1}{\varepsilon} \int_{\Omega} \tau_{22} \partial_{z} v_{2}}_{-\mathcal{D}_{2} / 2 r \nu}+\int_{\Omega} L_{2} v_{2}+\frac{1}{\varepsilon} \int_{\Omega} C_{2} v_{2} .
$$

Each term of $\int_{\Omega} L_{2} v_{2}$ and $\int_{\Omega} C_{2} v_{2}$ is estimated with the help of Poincare and Young inequalities as in the preceding step.

$$
\begin{aligned}
& \star \varepsilon^{2} \rho \int_{\Omega} \boldsymbol{v} \cdot \nabla u_{2}^{*} v_{2} \leq \varepsilon^{2} \underbrace{\rho C_{P}^{2}\left|\partial_{x} u_{2}^{*}\right|_{\infty}}_{=: M_{5}}\left(\frac{\varepsilon}{2}\left|\frac{1}{\varepsilon} \partial_{z} v_{1}\right|^{2}+\frac{\varepsilon}{2}\left|\partial_{z} v_{2}\right|^{2}\right)+\varepsilon^{2} \underbrace{\rho C_{P}^{2}\left|\partial_{z} u_{2}^{*}\right|_{\infty}}_{=: M_{6}}\left|\partial_{z} v_{2}\right|^{2} . \\
& \star \varepsilon^{2} \rho \int_{\Omega} \boldsymbol{u}^{*} \cdot \nabla v_{2} v_{2} \leq \varepsilon \underbrace{\rho C_{P}\left|u_{1}^{*}\right|_{\infty}}_{=: M_{7}}\left(\left|\varepsilon \partial_{x} v_{2}\right|^{2}+\left|\partial_{z} v_{2}\right|^{2}\right)+\varepsilon^{2} \underbrace{\rho C_{P}\left|u_{2}^{*}\right|_{\infty}}_{=: M_{8}}\left|\partial_{z} v_{2}\right|^{2} . \\
& \star \varepsilon^{2} \rho \int_{\Omega} \boldsymbol{u}^{*} \cdot \nabla u_{2}^{*} v_{2} \leq \frac{1}{2} \varepsilon^{2} \rho\left|\boldsymbol{u}^{*}\right|_{\infty}^{2}\left|\nabla u_{2}^{*}\right|^{2}+\varepsilon^{2} \underbrace{\frac{1}{2} C_{P}^{2}}_{=: M_{9}}\left|\partial_{z} v_{2}\right|^{2} \leq C+\varepsilon^{2} M_{9}\left|\partial_{z} v_{2}\right|^{2} .
\end{aligned}
$$

$\star$ By integration by parts (all boundary terms are equal to zero since $\left.v_{2}\right|_{\partial \Omega}=0$ ) and Young inequality as before:

$$
(1-r) \nu \varepsilon^{2} \int_{\Omega} \partial_{x}^{2} u_{2}^{*} v_{2}=-(1-r) \nu \varepsilon^{2} \int_{\Omega} \partial_{x} u_{2}^{*} \partial_{x} v_{2} \leq \varepsilon \underbrace{(1-r) \nu}_{=: M_{10}}\left(\frac{1}{2}\left|\partial_{x} u_{2}^{*}\right|^{2}+\frac{1}{2}\left|\varepsilon \partial_{x} v_{2}\right|^{2}\right) .
$$


$\star(1-r) \nu \int_{\Omega} \partial_{z}^{2} u_{2}^{*} r_{2} \leq \frac{1}{4 c_{1}}(1-r)^{2} \nu^{2} C_{P}^{2}\left|\partial_{z}^{2} u_{2}^{*}\right|^{2}+c_{1}\left|\partial_{z} v_{2}\right|^{2} \leq C+c_{1}\left|\partial_{z} v_{2}\right|^{2}$, where $c_{1}$ is a arbitrary positive constant.

$\star \int_{\Omega} \partial_{x} \sigma_{12}^{*} v_{2} \leq \frac{C_{P}^{2}}{4 c_{1}}\left|\partial_{x} \sigma_{12}^{*}\right|^{2}+c_{1}\left|\partial_{z} v_{2}\right|^{2} \leq C+c_{1}\left|\partial_{z} v_{2}\right|^{2}$

* The $C_{2}$ term is treated with integration by parts (again, no boundary terms since $\left.v_{2}\right|_{\partial \Omega}=$ $\left.\left.v_{1}\right|_{\partial \Omega}=0\right)$ and the divergence equation. The term is then treated as the preceding one:

$$
\begin{aligned}
\frac{1}{\varepsilon} \int_{\Omega} \partial_{z} \sigma_{22}^{*} v_{2}=-\frac{1}{\varepsilon} \int_{\Omega} \sigma_{22}^{*} \partial_{z} v_{2}=\frac{1}{\varepsilon} \int_{\Omega} \sigma_{22}^{*} \partial_{x} v_{1} & =-\frac{1}{\varepsilon} \int_{\Omega} \partial_{x} \sigma_{22}^{*} v_{1} \\
\leq C_{P}\left|\partial_{x} \sigma_{22}^{*}\right|\left|\frac{1}{\varepsilon} \partial_{z} v_{1}\right| & \leq \frac{C_{P}^{2}}{4 c_{2}}\left|\partial_{x} \sigma_{22}^{*}\right|^{2}+c_{2}\left|\frac{1}{\varepsilon} \partial_{z} v_{1}\right|^{2} \leq C+c_{2}\left|\frac{1}{\varepsilon} \partial_{z} v_{1}\right|^{2} .
\end{aligned}
$$

Now, let us choose $\varepsilon, c_{1}$ and $c_{2}$ small enough such that

$$
\frac{M_{5} \varepsilon^{3}}{2}, M_{6} \varepsilon^{2}, M_{7} \varepsilon, M_{8} \varepsilon, M_{9} \varepsilon, \frac{M_{10} \varepsilon}{2}, c_{1}, c_{2} \leq \frac{(1-r) \nu}{36} .
$$

Step 3. After summing (5.4) and (5.6), and multiplying by $2 r \nu$, it holds for $\varepsilon$ small enough (satisfying (5.5) and (5.7)):

$r \nu \rho \frac{\mathrm{d}}{\mathrm{d} t}\left(\left|v_{1}\right|^{2}+\left|\varepsilon v_{2}\right|^{2}\right)+\frac{3}{2} r(1-r) \nu^{2}\left(\left|\nabla_{\varepsilon} v_{1}\right|^{2}+\left|\varepsilon \nabla_{\varepsilon} v_{2}\right|^{2}\right)-\frac{2 r \nu}{\varepsilon^{2}} \int_{\Omega} q\left(\partial_{x} v_{1}+\partial_{z} v_{2}\right) \leq-\mathcal{D}_{1}-\mathcal{D}_{2}+C$,

where $C$ is a constant independent of $\varepsilon$. From the divergence equation $\nabla \cdot \boldsymbol{v}=\partial_{x} v_{1}+\partial_{z} v_{2}=0$ it follows that the pressure term $\int_{\Omega} q\left(\partial_{x} v_{1}+\partial_{z} v_{2}\right)=0$, and equation (5.3) is obtained.

\section{Proposition 5.3. Let us suppose that}

$$
\lambda^{*}\left|\partial_{z} u_{1}^{*}\right|_{\infty} \leq 1 / 12, \quad \lambda^{*}\left|\sigma_{12}^{*}\right|_{\infty} \leq \chi, \lambda^{*}\left(\left|\sigma_{11}^{*}\right|_{\infty}+\left|\sigma_{22}^{*}\right|_{\infty}\right) \leq \chi, 2 \lambda^{*}\left|\partial_{z} \sigma_{12}^{*}\right|_{\infty} \leq \chi, \quad \lambda^{*}\left|\partial_{z} \sigma_{11}^{*}\right|_{\infty} \leq \chi,
$$

where $\chi=\frac{\nu}{6} \sqrt{r(1-r)}$. Then for $\varepsilon$ small enough, $\tau_{11}, \tau_{12}, \tau_{22}$ solution of (5.1) satisfy the following inequality:

$$
\begin{aligned}
& \frac{\lambda^{*}}{2 \varepsilon} \frac{d}{d t}\left(\left|\tau_{11}\right|^{2}+2\left|\tau_{12}\right|^{2}+\left|\tau_{22}\right|^{2}\right)+\frac{1}{2}\left(\left|\frac{1}{\varepsilon} \tau_{11}\right|^{2}+2\left|\frac{1}{\varepsilon} \tau_{12}\right|^{2}+\left|\frac{1}{\varepsilon} \tau_{22}\right|^{2}\right) \\
& \quad+\frac{\eta}{\varepsilon}\left(\left|\nabla_{\varepsilon} \tau_{11}\right|^{2}+2\left|\nabla_{\varepsilon} \tau_{12}\right|^{2}+\left|\nabla_{\varepsilon} \tau_{22}\right|^{2}\right) \leq \mathcal{D}_{1}+\mathcal{D}_{2}+r(1-r) \nu^{2}\left(\left|\nabla_{\varepsilon} v_{1}\right|^{2}+\left|\varepsilon \nabla_{\varepsilon} v_{2}\right|^{2}\right)+C,
\end{aligned}
$$

where $C$ is a constant independent of $\varepsilon$.

Proof. As in the preceding proposition, classical a priori estimates on $\tau_{11}, \tau_{12}$ and $\tau_{22}$ are obtained, and the remaining terms are estimated accurately. 
Step 1. Let us multiply (5.1d) by $\frac{\tau_{11}}{\varepsilon}$ and integrate over $\Omega$. Again, the convection terms $\int_{\Omega} \boldsymbol{v} \cdot \nabla \tau_{11} \tau_{11}$ contained in $\int_{\Omega} \mathrm{d}_{t} \tau_{11} \tau_{11}$ are equal to zero by Lemma 5.1 , since $\nabla \cdot \boldsymbol{v}=0$ and $\left.\boldsymbol{v}\right|_{\partial \Omega}=0$ (see (5.2)). Moreover, there is no boundary term in the integration by parts since the boundary conditions on $\boldsymbol{\sigma}$ have be chosen such that $\left.\boldsymbol{\tau} \cdot n\right|_{\partial \Omega}=0$ (see also (5.2)). It follows:

$$
\begin{aligned}
\frac{\lambda^{*}}{2 \varepsilon} \frac{\mathrm{d}}{\mathrm{d} t}\left|\tau_{11}\right|^{2}-\frac{\lambda^{*}}{\varepsilon} \int_{\Omega} N\left(\boldsymbol{v}, \tau_{12}\right) \tau_{11} & +\left|\frac{1}{\varepsilon} \tau_{11}\right|^{2}+\frac{\eta}{\varepsilon}\left|\nabla_{\varepsilon} \tau_{11}\right|^{2} \\
& =\frac{2 r \nu}{\varepsilon} \int_{\Omega} \partial_{x} v_{1} \tau_{11}+\frac{1}{\varepsilon} \int_{\Omega} L_{11} \tau_{11}+\frac{1}{\varepsilon^{2}} \int_{\Omega} L_{11}^{\prime} \tau_{11} .
\end{aligned}
$$

- The terms of $\int_{\Omega} \mathcal{L}_{11} \tau_{11}$ are estimated as follows:

$\star \lambda^{*} \int_{\Omega} \partial_{x} u_{2}^{*} \tau_{12} \tau_{11} \leq \underbrace{\lambda^{*}\left|\partial_{x} u_{2}^{*}\right|_{\infty}}_{=: M_{11}}\left(\frac{\varepsilon^{2}}{2}\left|\frac{1}{\varepsilon} \tau_{11}\right|^{2}+\frac{\varepsilon^{2}}{2}\left|\frac{1}{\varepsilon} \tau_{12}\right|^{2}\right)$.

$\star$ In a same way: $\frac{\lambda^{*}}{\varepsilon} \int_{\Omega} v_{1} \partial_{x} \sigma_{11}^{*} \tau_{11} \leq \underbrace{\lambda^{*}\left|\partial_{x} \sigma_{11}^{*}\right|_{\infty} C_{P}}_{=: M_{12}}\left(\frac{\varepsilon}{2}\left|\frac{1}{\varepsilon} \partial_{z} v_{1}\right|^{2}+\frac{\varepsilon}{2}\left|\frac{1}{\varepsilon} \tau_{11}\right|^{2}\right)$.

Let us choose $\varepsilon$ small enough such that:

$$
\begin{gathered}
\frac{M_{11} \varepsilon^{2}}{2} \leq \frac{1}{24} \quad \text { and } \quad \frac{M_{12} \varepsilon}{2} \leq \operatorname{Min}\left\{\frac{r(1-r) \nu}{6}, \frac{1}{24}\right\} . \\
\star \lambda^{*} \int_{\Omega} \partial_{x} v_{2} \sigma_{12}^{*} \tau_{11} \leq \lambda^{*}\left|\sigma_{12}^{*}\right|_{\infty}\left|\varepsilon \partial_{x} v_{2}\right|\left|\frac{1}{\varepsilon} \tau_{11}\right| \leq \lambda^{*}\left|\sigma_{12}^{*}\right|_{\infty}\left(\frac{1}{4 c_{3}}\left|\varepsilon \partial_{x} v_{2}\right|^{2}+c_{3}\left|\frac{1}{\varepsilon} \tau_{11}\right|^{2}\right) .
\end{gathered}
$$

Here, it is not possible to choose $c_{3}$ such that both coefficients are less than $r(1-r) \nu / 6$ and 1/24. Therefore, a condition on $\lambda^{*}\left|\sigma_{12}^{*}\right|_{\infty}$ is imposed such that:

$$
\frac{\lambda^{*}\left|\sigma_{12}^{*}\right|_{\infty}}{4 c_{3}} \leq \frac{r(1-r) \nu}{6} \quad \text { and } \quad \lambda^{*}\left|\sigma_{12}^{*}\right|_{\infty} c_{3} \leq \frac{1}{24}
$$

Choosing $c_{3}$ satisfying $\lambda^{*}\left|\sigma_{12}^{*}\right|_{\infty} c_{3}=1 / 24$, the condition on $\lambda^{*}\left|\sigma_{12}^{*}\right|_{\infty}$ becomes:

$$
\lambda^{*}\left|\sigma_{12}^{*}\right|_{\infty} \leq \frac{\nu}{6} \sqrt{r(1-r)}=: \chi
$$

* Similarly the following term can be estimated:

$$
\frac{\lambda^{*}}{\varepsilon} \int_{\Omega} v_{2} \partial_{z} \sigma_{11}^{*} \tau_{11} \leq \lambda^{*}\left|\partial_{z} \sigma_{11}^{*}\right|_{\infty}\left|\partial_{z} v_{2}\right|\left|\frac{1}{\varepsilon} \tau_{11}\right| \leq \lambda^{*}\left|\partial_{z} \sigma_{11}^{*}\right|_{\infty}\left(\frac{1}{4 c_{3}}\left|\partial_{z} v_{2}\right|^{2}+c_{3}\left|\frac{1}{\varepsilon} \tau_{11}\right|^{2}\right) \text {. }
$$

The same reasoning as before allows to control both terms providing that $\lambda^{*}\left|\partial_{z} \sigma_{11}^{*}\right|_{\infty} \leq \chi$.

$\star$ In order to treat the term $-\lambda^{*} \int_{\Omega} \boldsymbol{u}^{*} \cdot \nabla \tau_{11} \tau_{11}$, it is not possible to apply Lemma 5.1 , since 
$\left.\boldsymbol{u}^{*} \cdot n\right|_{\partial \Omega} \neq 0$. However, integration by parts implies that

$$
-\lambda^{*} \int_{\Omega} \boldsymbol{u}^{*} \cdot \nabla \tau_{11} \tau_{11}=-\frac{\lambda^{*}}{2} \int_{\partial \Omega} \boldsymbol{u}^{*} \cdot n \tau_{11}^{2}
$$

On $\omega$, since $\boldsymbol{u}^{*}=(s, 0)$ (see $(4.2)$ ), it holds $\boldsymbol{u}^{*} \cdot n=0$. Thus it remains to consider the boundary integral on $\Gamma_{L}$. This boundary integral is split into two integrals on $\Gamma_{+}$and $\Gamma_{-}$. On $\Gamma_{-}$, it holds $\boldsymbol{u}^{*} \cdot n>0$, thus $-\frac{\lambda^{*}}{2} \int_{\Gamma_{-}} \boldsymbol{u}^{*} \cdot n \tau_{11}^{2} \leq 0$, and this term is trivially bounded by zero. On $\Gamma_{+}$, the boundary conditions are chosen in subsection 4.2 such that $\left.\boldsymbol{\tau}\right|_{\Gamma_{+}}=0$, therefore $-\frac{\lambda^{*}}{2} \int_{\Gamma_{+}} \boldsymbol{u}^{*} \cdot n \tau_{11}^{2}=0$.

- All other terms of $\int_{\Omega} L_{11} \tau_{11}$ are easier to manage, since they are linear in $\tau_{11}$, and they are treated with Young and Poincaré inequalities in a same way as the ones in $v_{1}, v_{2}$.

- For the terms of $\int_{\Omega} L_{11}^{\prime} \tau_{11}$, we proceed as before:

$$
\frac{\lambda^{*}}{\varepsilon^{2}} \int_{\Omega} \partial_{z} u_{1}^{*} \tau_{12} \tau_{11} \leq \lambda^{*}\left|\partial_{z} u_{1}^{*}\right|_{\infty}\left|\frac{1}{\varepsilon} \tau_{12}\right|\left|\frac{1}{\varepsilon} \tau_{11}\right| \leq \lambda^{*}\left|\partial_{z} u_{1}^{*}\right|_{\infty}\left(\frac{1}{2}\left|\frac{1}{\varepsilon} \tau_{12}\right|^{2}+\frac{1}{2}\left|\frac{1}{\varepsilon} \tau_{11}\right|^{2}\right) .
$$

Choosing $\lambda^{*}\left|\partial_{z} u_{1}^{*}\right|_{\infty} \leq 1 / 12$, both terms are bounded by $1 / 24$.

$$
\frac{\lambda^{*}}{\varepsilon^{2}} \int_{\Omega} \partial_{z} v_{1} \sigma_{12}^{*} \tau_{11} \leq \lambda^{*}\left|\sigma_{12}^{*}\right|_{\infty}\left|\frac{1}{\varepsilon} \partial_{z} v_{1}\right|\left|\frac{1}{\varepsilon} \tau_{11}\right| \leq \lambda^{*}\left|\sigma_{12}^{*}\right|_{\infty}\left(\frac{1}{4 c_{3}}\left|\frac{1}{\varepsilon} \partial_{z} v_{1}\right|^{2}+c_{3}\left|\frac{1}{\varepsilon} \tau_{11}\right|^{2}\right) .
$$

Imposing $\lambda^{*}\left|\sigma_{12}^{*}\right|_{\infty} \leq \chi$ is enough to ensure that the coefficients are less than $r(1-r) \nu / 6$ and $1 / 24$.

Step 2. Now, multiplying equation (5.1e) by $\frac{2 \tau_{12}}{\varepsilon}$ and integrating over $\Omega$, with the same reasoning as in the preceding step it follows:

$$
\begin{aligned}
\frac{\lambda^{*}}{\varepsilon} \frac{\mathrm{d}}{\mathrm{d} t}\left|\tau_{12}\right|^{2}+\frac{\lambda^{*}}{\varepsilon} & \int_{\Omega} N\left(\boldsymbol{v}, \tau_{11}-\tau_{22}\right) \tau_{12}+2\left|\frac{1}{\varepsilon} \tau_{12}\right|^{2}+\frac{2 \eta}{\varepsilon}\left|\nabla_{\varepsilon} \tau_{12}\right|^{2} \\
& =\frac{2 r \nu}{\varepsilon} \int_{\Omega}\left(\partial_{x} v_{2}+\frac{1}{\varepsilon} \partial_{z} v_{1}\right) \tau_{12}+\frac{2}{\varepsilon} \int_{\Omega} L_{12} \tau_{12}+\frac{2}{\varepsilon^{2}} \int_{\Omega} L_{12}^{\prime} \tau_{12}
\end{aligned}
$$

The terms in $L_{12}$ and $L_{12}^{\prime}$ are of the same type as the ones in $L_{11}$ and $L_{11}^{\prime}$, and are treated very similarly to them, applying Young inequality, and assuming smallness assumptions on $\varepsilon$. Thus, let us only write the terms needing additional assumptions.

$\star \lambda^{*} \int_{\Omega} \partial_{x} v_{2}\left(\sigma_{11}^{*}-\sigma_{22}^{*}\right) \tau_{12} \leq \lambda^{*}\left(\left|\sigma_{11}^{*}\right|_{\infty}+\left|\sigma_{22}^{*}\right|_{\infty}\right)\left|\varepsilon \partial_{x} v_{2}\right|\left|\frac{1}{\varepsilon} \tau_{12}\right|$, and it is enough to assume that $\lambda^{*}\left(\left|\sigma_{11}^{*}\right|_{\infty}+\left|\sigma_{22}^{*}\right|_{\infty}\right) \leq \chi$. 
$\star \frac{2 \lambda^{*}}{\varepsilon} \int_{\Omega} v_{2} \partial_{z} \sigma_{12}^{*} \tau_{12} \leq 2 \lambda^{*}\left|\partial_{z} \sigma_{12}^{*}\right|_{\infty}\left|\partial_{z} v_{2}\right|\left|\frac{1}{\varepsilon} \tau_{12}\right|$, and we assume that $2 \lambda^{*}\left|\partial_{z} \sigma_{12}^{*}\right|_{\infty} \leq \chi$.

$\star \frac{\lambda^{*}}{\varepsilon^{2}} \int_{\Omega} \partial_{z} u^{*}\left(\tau_{11}-\tau_{22}\right) \tau_{12} \leq \lambda^{*}\left|\partial_{z} u_{1}^{*}\right|_{\infty}\left(\left|\frac{1}{\varepsilon} \tau_{11}\right|+\left|\frac{1}{\varepsilon} \tau_{22}\right|\right)\left|\frac{1}{\varepsilon} \tau_{12}\right|$, it has already been assumed that $\lambda^{*}\left|\partial_{z} u_{1}^{*}\right|_{\infty} \leq 1 / 12$.

$\star \frac{\lambda^{*}}{\varepsilon^{2}} \int_{\Omega} \partial_{z} v_{1}\left(\sigma_{11}^{*}-\sigma_{22}^{*}\right) \tau_{12} \leq \lambda^{*}\left(\left|\sigma_{11}^{*}\right|_{\infty}+\left|\sigma_{22}^{*}\right|_{\infty}\right)\left|\frac{1}{\varepsilon} \partial_{z} v_{1}\right|\left|\frac{1}{\varepsilon} \tau_{12}\right|$, it has already been assumed that $\lambda^{*}\left(\left|\sigma_{11}^{*}\right|_{\infty}+\left|\sigma_{22}^{*}\right|_{\infty}\right) \leq \chi$.

Step 3. Multiplying (5.1f) by $\frac{\tau_{22}}{\varepsilon}$, and estimating the terms just as the ones in $\tau_{11}$, it follows

$$
\begin{aligned}
\frac{\lambda^{*}}{2 \varepsilon} \frac{\mathrm{d}}{\mathrm{d} t}\left|\tau_{22}\right|^{2}+\frac{\lambda^{*}}{\varepsilon} \int_{\Omega} N\left(\boldsymbol{v}, \tau_{12}\right) \tau_{22} & +\left|\frac{1}{\varepsilon} \tau_{22}\right|^{2}+\frac{\eta}{\varepsilon}\left|\nabla_{\varepsilon} \tau_{22}\right|^{2} \\
& =\frac{2 r \nu}{\varepsilon^{2}} \int_{\Omega} \partial_{z} v_{2} c+\frac{1}{\varepsilon} \int_{\Omega} L_{22} \tau_{22}+\frac{1}{\varepsilon^{2}} \int_{\Omega} L_{22}^{\prime} \tau_{22} .
\end{aligned}
$$

Assuming that $\lambda\left|\sigma_{12}^{*}\right|_{\infty} \leq \chi, \lambda^{*}\left|\partial_{z} \sigma_{11}^{*}\right|_{\infty} \leq \chi$ and $\lambda^{*}\left|\partial_{z} u_{1}^{*}\right|_{\infty} \leq 1 / 12$, all the terms $\frac{1}{\varepsilon} \int_{\Omega} L_{22} \tau_{22}$ and $\frac{1}{\varepsilon^{2}} \int_{\Omega} L_{22}^{\prime} \tau_{22}$ are bounded and estimated as in Step 1.

Step 4. Summing (5.9), (5.10) and (5.11), and noticing that

$$
\begin{aligned}
-\int_{\Omega} N\left(\boldsymbol{v}, \tau_{12}\right) \tau_{11}+ & \int_{\Omega} N\left(\boldsymbol{v}, \tau_{11}-\tau_{22}\right) \tau_{12}+\int_{\Omega} N\left(\boldsymbol{v}, \tau_{12}\right) \tau_{22} \\
& =\int_{\Omega}\left(\varepsilon \partial_{x} v_{2}-\frac{1}{\varepsilon} \partial_{z} v_{1}\right)\left(-\tau_{12} \tau_{11}+\left(\tau_{11}-\tau_{22}\right) \tau_{12}+\tau_{12} \tau_{22}\right)=0,
\end{aligned}
$$

it follows that for $\varepsilon$ small enough

$$
\begin{aligned}
\frac{\lambda^{*}}{2 \varepsilon} \frac{\mathrm{d}}{\mathrm{d} t}\left(\left|\tau_{11}\right|^{2}+2\left|\tau_{12}\right|^{2}+\left|\tau_{22}\right|^{2}\right) & +\frac{1}{2}\left(\left|\frac{1}{\varepsilon} \tau_{11}\right|^{2}+2\left|\frac{1}{\varepsilon} \tau_{12}\right|^{2}+\left|\frac{1}{\varepsilon} \tau_{22}\right|^{2}\right)+\frac{\eta}{\varepsilon}\left(\left|\nabla_{\varepsilon} \tau_{11}\right|^{2}+2\left|\nabla_{\varepsilon} \tau_{12}\right|^{2}+\left|\nabla_{\varepsilon} \tau_{22}\right|^{2}\right) \\
& \leq \mathcal{D}_{1}+\mathcal{D}_{2}+r(1-r) \nu^{2}\left(\left|\nabla_{\varepsilon} v_{1}\right|^{2}+\left|\varepsilon \nabla_{\varepsilon} v_{2}\right|^{2}\right)+C
\end{aligned}
$$

where we recognized the terms $\mathcal{D}_{1}+\mathcal{D}_{2}$, and where $C$ is a constant independent of $\varepsilon$.

From now on, let us come back to the notation with the superscripts ${ }^{\varepsilon \eta}$, denoting the dependence on $\varepsilon$ and $\eta$.

Theorem 5.4. Suppose that the solution $\boldsymbol{u}^{*}, \boldsymbol{\sigma}^{*}$ of system (4.1)-(4.2) satisfies the following small- 
ness assumptions

$$
\lambda^{*}\left|\partial_{z} u_{1}^{*}\right|_{\infty} \leq 1 / 12, \quad \lambda^{*}\left|\sigma_{12}^{*}\right|_{\infty} \leq \chi, \lambda^{*}\left(\left|\sigma_{11}^{*}\right|_{\infty}+\left|\sigma_{22}^{*}\right|_{\infty}\right) \leq \chi, 2 \lambda^{*}\left|\partial_{z} \sigma_{12}^{*}\right|_{\infty} \leq \chi, \quad \lambda^{*}\left|\partial_{z} \sigma_{11}^{*}\right|_{\infty} \leq \chi,
$$

where $\chi=\frac{\nu}{6} \sqrt{r(1-r)}$. Then the following convergences hold up to subsequences when $\eta$ and then $\varepsilon$ tend to zero:

$$
\begin{array}{r}
u_{1}^{\varepsilon \eta} \rightarrow u_{1}^{*}, \quad \partial_{z} u_{1}^{\varepsilon \eta} \rightarrow \partial_{z} u_{1}^{*}, \quad \partial_{x} u_{1}^{\varepsilon \eta} \rightarrow \partial_{x} u_{1}^{*} \quad \text { in } L^{2}\left(0, T, L^{2}(\Omega)\right), \\
u_{2}^{\varepsilon \eta} \rightarrow 0, \quad \partial_{z} u_{2}^{\varepsilon \eta} \rightarrow 0, \quad \partial_{x} u_{2}^{\varepsilon \eta} \rightarrow 0 \quad \text { in } L^{2}\left(0, T, L^{2}(\Omega)\right), \\
\varepsilon \boldsymbol{\sigma}^{\varepsilon \eta} \rightarrow \boldsymbol{\sigma}^{*} \quad \text { in } L^{2}\left(0, T, L^{2}(\Omega)\right), \\
u_{1}^{\varepsilon \eta} \rightarrow^{*} u_{1}^{*}, \quad u_{2}^{\varepsilon \eta} \rightarrow^{*} 0, \quad \varepsilon \boldsymbol{\sigma}^{\varepsilon \eta} \rightarrow \boldsymbol{\sigma}^{*} \quad \text { in } L^{\infty}\left(0, T, L^{2}(\Omega)\right) .
\end{array}
$$

Proof. Summing (5.3), (5.8) implies that for $\varepsilon$ small enough (i.e. if assumption (5.12) is satisfied):

$$
\begin{aligned}
& r \nu \rho \frac{\mathrm{d}}{\mathrm{d} t}\left(\left|v_{1}^{\varepsilon \eta}\right|^{2}+\left|\varepsilon v_{2}^{\varepsilon \eta}\right|^{2}\right)+\frac{\lambda^{*}}{2 \varepsilon} \frac{\mathrm{d}}{\mathrm{d} t}\left(\left|\tau_{11}^{\varepsilon \eta}\right|^{2}+2\left|\tau_{12}^{\varepsilon \eta}\right|^{2}+\left|\tau_{22}^{\varepsilon \eta}\right|^{2}\right)+\frac{\eta}{\varepsilon}\left(\left|\nabla_{\varepsilon} \tau_{11}^{\varepsilon \eta}\right|^{2}+2\left|\nabla_{\varepsilon} \tau_{12}^{\varepsilon \eta}\right|^{2}+\left|\nabla_{\varepsilon} \tau_{22}^{\varepsilon \eta}\right|^{2}\right) \\
& +\frac{r(1-r) \nu^{2}}{2}\left(\left|\partial_{x} v_{1}^{\varepsilon \eta}\right|^{2}+\left|\frac{1}{\varepsilon} \partial_{z} v_{1}^{\varepsilon \eta}\right|^{2}+\left|\varepsilon \partial_{x} v_{2}^{\varepsilon \eta}\right|^{2}+\left|\partial_{z} v_{2}^{\varepsilon \eta}\right|^{2}\right)+\frac{1}{2}\left|\frac{1}{\varepsilon} \tau_{11}^{\varepsilon \eta}\right|^{2}+\left|\frac{1}{\varepsilon} \tau_{12}^{\varepsilon \eta}\right|^{2}+\frac{1}{2}\left|\frac{1}{\varepsilon} \tau_{22}^{\varepsilon \eta}\right|^{2} \leq C .
\end{aligned}
$$

From this inequality, it follows that $\boldsymbol{v}^{\varepsilon \eta}$ converges to $\boldsymbol{v}^{\varepsilon}$ in $\boldsymbol{H}^{1}(\Omega)$ and $\boldsymbol{\tau}^{\varepsilon \eta}$ converges $\boldsymbol{\tau}^{\varepsilon}$ in $\boldsymbol{L}^{2}(\Omega)$, as $\eta$ tends to zero. $\boldsymbol{v}^{\varepsilon}$ and $\boldsymbol{\tau}^{\varepsilon}$ are the solutions solutions of (5.1) without the terms $\eta \Delta \boldsymbol{\tau}^{\varepsilon \eta}$. Indeed, recalling the weak formulation of the system (5.1), it suffices to notice that Hölder's inequality allows to treat the term $\eta \Delta \boldsymbol{\tau}^{\varepsilon \eta}$ :

$$
\eta \int_{\Omega} \nabla_{\varepsilon} \boldsymbol{\tau}^{\varepsilon \eta} \cdot \nabla_{\varepsilon} \boldsymbol{\phi} \leq \eta^{1 / 2}(\underbrace{\eta\left|\nabla_{\varepsilon} \boldsymbol{\tau}^{\varepsilon \eta}\right|^{2}}_{\leq C}+\left|\nabla_{\varepsilon} \boldsymbol{\phi}\right|^{2}) \underset{\eta \rightarrow 0}{\longrightarrow} 0, \quad \forall \boldsymbol{\phi} \in \boldsymbol{H}_{0}^{1}(\Omega) .
$$

Moreover, $\boldsymbol{v}^{\varepsilon}$ and $\boldsymbol{\tau}^{\varepsilon}$ satisfy the following estimate:

$$
\begin{aligned}
& r \nu \rho \frac{\mathrm{d}}{\mathrm{d} t}\left(\left|v_{1}^{\varepsilon}\right|^{2}+\left|\varepsilon v_{2}^{\varepsilon}\right|^{2}\right)+\frac{\lambda^{*}}{2 \varepsilon} \frac{\mathrm{d}}{\mathrm{d} t}\left(\left|\tau_{11}^{\varepsilon}\right|^{2}+2\left|\tau_{12}^{\varepsilon}\right|^{2}+\left|\tau_{22}^{\varepsilon}\right|^{2}\right) \\
& +\frac{1}{2} r(1-r) \nu^{2}\left(\left|\partial_{x} v_{1}^{\varepsilon}\right|^{2}+\left|\frac{1}{\varepsilon} \partial_{z} v_{1}^{\varepsilon}\right|^{2}+\left|\varepsilon \partial_{x} v_{2}^{\varepsilon}\right|^{2}+\left|\partial_{z} v_{2}^{\varepsilon}\right|^{2}\right)+\frac{1}{2}\left|\frac{1}{\varepsilon} \tau_{11}^{\varepsilon}\right|^{2}+\left|\frac{1}{\varepsilon} \tau_{12}^{\varepsilon}\right|^{2}+\frac{1}{2}\left|\frac{1}{\varepsilon} \tau_{22}^{\varepsilon}\right|^{2} \leq C .
\end{aligned}
$$

It remains to pass to the limit as $\varepsilon$ tends to zero. After integrating (5.18) between 0 and $T$, it yields that

$\triangleright\left\|v_{1}^{\varepsilon}\right\|_{L^{2}\left(L^{2}\right)} \leq\left\|\partial_{z} v_{1}^{\varepsilon}\right\|_{L^{2}\left(L^{2}\right)} \leq C \varepsilon$, thus the following convergences hold in $L^{2}\left(0, T, L^{2}(\Omega)\right)$ as $\varepsilon$ tends to zero:

$$
v_{1}^{\varepsilon} \rightarrow 0 \quad \text { and } \quad \partial_{z} v_{1}^{\varepsilon} \rightarrow 0 .
$$

From these convergences, it follows that $u_{1}^{\varepsilon}=u_{1}^{*}+v_{1}^{\varepsilon} \rightarrow u_{1}^{*}$ in $L^{2}\left(0, T, L^{2}(\Omega)\right)$ and $\partial_{z} u_{1}^{\varepsilon} \rightarrow \partial_{z} u_{1}^{*}$ 
in $L^{2}\left(0, T, L^{2}(\Omega)\right)$.

$\triangleright\left\|\partial_{x} v_{1}^{\varepsilon}\right\|_{L^{2}\left(L^{2}\right)} \leq C$, thus $\partial_{x} v_{1}^{\varepsilon}$ converges weakly in $L^{2}\left(0, T, L^{2}(\Omega)\right)$. Now, since it is already known that $u_{1}^{\varepsilon} \rightarrow u_{1}^{*}$, it follows that $\partial_{x} u_{1}^{\varepsilon} \rightarrow \partial_{x} u_{1}^{*}$ in $L^{2}\left(0, T, L^{2}(\Omega)\right)$.

$\triangleright$ Similarly $\left\|v_{2}^{\varepsilon}\right\|_{L^{2}\left(L^{2}\right)} \leq\left\|\partial_{z} v_{2}^{\varepsilon}\right\|_{L^{2}\left(L^{2}\right)} \leq C$, thus $\varepsilon v_{2}^{\varepsilon}$ and $\varepsilon \partial_{z} v_{2}^{\varepsilon}$ converge strongly to zero in $L^{2}\left(0, T, L^{2}(\Omega)\right)$, and thus $u_{2}^{\varepsilon}=\varepsilon u_{2}^{*}+\varepsilon v_{2}^{\varepsilon} \rightarrow 0$ in $L^{2}\left(0, T, L^{2}(\Omega)\right)$, and $\partial_{z} u_{2}^{\varepsilon} \rightarrow 0$ in $L^{2}\left(0, T, L^{2}(\Omega)\right)$.

$\triangleright\left\|\partial_{x} v_{2}^{\varepsilon}\right\|_{L^{2}\left(L^{2}\right)} \leq \frac{C}{\varepsilon}$, thus $\partial_{x} u_{2}^{\varepsilon}$ converges weakly in $L^{2}\left(0, T, L^{2}(\Omega)\right)$. Since $u_{2}^{\varepsilon} \rightarrow 0$, it implies that $\partial_{x} u_{2}^{\varepsilon} \rightarrow 0$ in $L^{2}\left(0, T, L^{2}(\Omega)\right)$.

$\triangleright\left\|\tau_{11}^{\varepsilon}\right\|_{L^{2}\left(L^{2}\right)},\left\|\tau_{12}^{\varepsilon}\right\|_{L^{2}\left(L^{2}\right)},\left\|\tau_{22}^{\varepsilon}\right\|_{L^{2}\left(L^{2}\right)} \leq C \varepsilon$, therefore $\tau_{11}^{\varepsilon}, \tau_{12}^{\varepsilon}, \tau_{22}^{\varepsilon} \rightarrow 0$ in $L^{2}\left(0, T, L^{2}(\Omega)\right)$. Thus $\varepsilon \sigma_{11}^{\varepsilon}=\sigma_{11}^{*}+\tau_{11}^{\varepsilon} \rightarrow \sigma_{11}^{*}$ in $L^{2}\left(0, T, L^{2}(\Omega)\right)$, and in the same way $\varepsilon \sigma_{12}^{\varepsilon} \rightarrow \sigma_{12}^{*}$ in $L^{2}\left(0, T, L^{2}(\Omega)\right)$, $\varepsilon \sigma_{22}^{\varepsilon} \rightarrow \sigma_{22}^{*}$ in $L^{2}\left(0, T, L^{2}(\Omega)\right)$.

$\triangleright$ From the terms with the derivatives in time, using the fact that $\left.\boldsymbol{v}^{\varepsilon}\right|_{t=0}=\boldsymbol{u}_{0}^{\varepsilon}-\boldsymbol{u}^{*} \in \boldsymbol{L}^{2}(\Omega)$ and $\left.\boldsymbol{\tau}^{\varepsilon}\right|_{t=0}=\boldsymbol{\sigma}_{0}^{\varepsilon}-\boldsymbol{\sigma}^{*} \in \boldsymbol{L}^{2}(\Omega)$ are bounded independently of $\varepsilon$, we can conclude that

$$
\left\|\boldsymbol{v}^{\varepsilon}\right\|_{L^{\infty}\left(L^{2}\right)} \leq C \quad \text { and } \quad\left\|\boldsymbol{\tau}^{\varepsilon}\right\|_{L^{\infty}\left(L^{2}\right)} \leq C \sqrt{\varepsilon}
$$

These estimates and the uniqueness of the limit imply that $v_{1}^{\varepsilon}$ and $\varepsilon v_{2}^{\varepsilon}$ converge weakly-* in $L^{\infty}\left(0, T, L^{2}(\Omega)\right)$ toward zero, and that $\boldsymbol{\tau}^{\varepsilon}$ converges strongly in $L^{\infty}\left(0, T, \boldsymbol{L}^{2}(\Omega)\right)$ toward zero, which proves the last estimate (5.16).

Note that in a simplified case (with a simpler geometry), the hypothesis (5.12) is satisfied under a small data assumption on the physical parameters.

Remark 5.5. When $h$ is constant with respect to $x, p^{*}$ is also independent of $x$, so that equation (4.1) reduces to

$$
-(1-r) \partial_{z}^{2} u_{1}^{*}-r \frac{\partial}{\partial z}\left(\frac{\partial_{z} u_{1}^{*}}{1+\lambda^{* 2}\left|\partial_{z} u_{1}^{*}\right|^{2}}\right)=0 .
$$

It has been shown in [8] for example that for $r<8 / 9$ this equation admits a unique solution $u_{1}^{*}=s\left(1-\frac{z}{h}\right)$.

Now, it follows that $\sigma_{12}^{*}=\frac{r \nu \partial_{z} u_{1}^{*}}{1+\lambda^{* 2}\left|\partial_{z} u_{1}^{*}\right|^{2}}=\frac{-r \nu s}{h+\lambda^{* 2} s^{2} / h}$, and $\sigma_{11}^{*}=-\sigma_{22}^{*}=-\lambda^{*} \partial_{z} u_{1}^{*} \sigma_{12}^{*}=$ $\frac{-r \nu s^{2} \lambda^{*}}{h^{2}+\lambda^{* 2} s^{2}}$.

In this case, hypothesis (5.12) becomes more simple. Since $\partial_{z} u_{1}^{*}=-s / h, \sigma_{11}^{*}$ and $\sigma_{12}^{*}$ are constant with respect to $z$, so that the last two conditions are trivially verified. Using the fact that $r<8 / 9$, it leads to a smallness condition on $s \lambda^{*}$ with respect to $h\left(s \lambda^{*} \leq h / 12\right.$ is enough in order to satisfy all conditions).

Observe that this condition is not optimal, but it shows that in the simplified case when $h(x)$ is constant, a simple choice of the parameters $s, \lambda^{*}$ and $h$ satisfies hypothesis (5.12).

\subsection{Convergence of the pressure}

It remains to prove the convergence of the pressure. 
Theorem 5.6. Under the same smallness assumption (5.12), the following convergence result holds up to a subsequence for $p$ :

$$
\varepsilon^{2} p \underset{\varepsilon \rightarrow 0}{\rightarrow} p^{*} \quad \text { in } \mathcal{D}^{\prime}\left(0, T, L^{2}(\Omega)\right)
$$

Proof. Throughout the proof, $C$ will denote some generic constants independent of $\varepsilon$. Let $\varepsilon \leq 1$. Let us integrate over $\Omega_{T}=\Omega \times(0, T)$ equation (5.1a) multiplied by $\varepsilon^{2} \varphi_{1}$, for any function $\phi_{1} \in$ $H_{0}^{1}(\Omega)$. It follows:

$$
\begin{gathered}
\rho \varepsilon^{2} \int_{\Omega_{T}} \partial_{t} v_{1} \phi_{1}+\rho \varepsilon^{2} \int_{\Omega_{T}} v_{1} \partial_{x} v_{1} \phi_{1}+\rho \varepsilon \int_{\Omega_{T}} v_{2} \partial_{z} v_{1} \phi_{1}+(1-r) \nu \varepsilon^{2} \int_{\Omega_{T}} \partial_{x} v_{1} \partial_{x} \phi_{1}+(1-r) \nu \int_{\Omega_{T}} \partial_{z} v_{1} \partial_{z} \phi_{1} \\
\quad+\int_{\Omega_{T}} \partial_{x} q \phi_{1}=-\varepsilon \int_{\Omega_{T}} \tau_{11} \partial_{x} \phi_{1}-\int_{\Omega_{T}} \tau_{12} \partial_{z} \phi_{1}+\varepsilon^{2} \int_{\Omega_{T}} L_{1} \phi_{1}+\varepsilon \int_{\Omega_{T}} C_{1} \phi_{1}, \quad \forall \phi_{1} \in H_{0}^{1}(\Omega) .
\end{gathered}
$$

Using the fact that $\phi_{1}$ is independent of $t$, the first term becomes

$$
\rho \varepsilon^{2} \int_{\Omega_{T}} \partial_{t} v_{1} \phi_{1}=\rho \varepsilon^{2} \int_{\Omega} \phi_{1} \int_{0}^{T} \partial_{t} v_{1}=\rho \varepsilon^{2} \int_{\Omega} \phi_{1}\left(v_{1}(T)-v_{1}(0)\right),
$$

where $v_{1}(0)=u_{1_{0}}-u_{1}^{*}$ denotes the value of $v_{1}$ at time $t=0$. Now, introducing

$$
\pi=\int_{0}^{T} q \mathrm{~d} t
$$

and using integration by parts for the pressure term (the boundary term is zero since $\phi_{1} \in H_{0}^{1}(\Omega)$ ), (5.21) becomes: $\forall \phi_{1} \in H_{0}^{1}(\Omega)$,

$$
\begin{aligned}
& \rho \varepsilon^{2} \int_{\Omega} \phi_{1}\left(v_{1}(T)-v_{1}(0)\right)+\rho \varepsilon^{2} \int_{\Omega_{T}} v_{1} \partial_{x} v_{1} \phi_{1}+\rho \varepsilon \int_{\Omega_{T}} r_{2} \partial_{z} v_{1} \phi_{1}+(1-r) \nu \varepsilon^{2} \int_{\Omega_{T}} \partial_{x} v_{1} \partial_{x} \phi_{1} \\
& \quad+(1-r) \nu \int_{\Omega_{T}} \partial_{z} v_{1} \partial_{z} \phi_{1}-\int_{\Omega} \pi \partial_{x} \phi_{1}=-\varepsilon \int_{\Omega_{T}} \tau_{11} \partial_{x} \phi_{1}-\int_{\Omega_{T}} \tau_{12} \partial_{z} \phi_{1}+\varepsilon^{2} \int_{\Omega_{T}} L_{1} \phi_{1}+\varepsilon \int_{\Omega_{T}} C_{1} \phi_{1} .
\end{aligned}
$$

It remains to estimate all terms independent of $\pi$. The non-linear terms are to bee handled with care, since $\phi_{1} \notin L^{\infty}(\Omega)$. Proceeding as in [3], Hölder inequality with exponents $2+\delta, \delta^{\prime}$ and 2 leads:

$$
\left|\int_{\Omega_{T}} v_{1} \partial_{x} v_{1} \phi_{1}\right| \leq\left|\phi_{1}\right|_{\delta^{\prime}} \int_{0}^{T}\left|v_{1}\right|_{2+\delta}\left|\partial_{x} v_{1}\right|
$$


where $\frac{1}{2+\delta}+\frac{1}{2}+\frac{1}{\delta^{\prime}}=1$ (which implies that $\delta^{\prime}=\frac{2(2+\delta)}{\delta}$ ). According to interpolation theory, $\left[L^{2}, L^{4}\right]_{\theta}=L^{2+\delta}$ for $\theta=\frac{\delta}{2+\delta}$, and the following estimate holds:

$$
\left|v_{1}\right|_{2+\delta} \leq C\left|v_{1}\right|_{4}^{\theta}\left|v_{1}\right|^{1-\theta}
$$

Moreover Lemma 3.2 of [1] states that for $v_{1} \in H_{0}^{1}(\Omega)$, it holds:

$$
\left|v_{1}\right|_{4} \leq \sqrt{2}\left|\partial_{x} v_{1}\right|^{1 / 4}\left|\partial_{z} v_{1}\right|^{3 / 4}
$$

Using the two last inequalities and Poincaré inequality, (5.22) becomes

$$
\rho \varepsilon^{2}\left|\int_{\Omega_{T}} v_{1} \partial_{x} v_{1} \phi_{1}\right| \leq \rho \varepsilon^{2}\left|\phi_{1}\right| \delta_{\delta^{\prime}} C \int_{0}^{T}\left|\partial_{x} v_{1}\right|^{\theta / 4}\left|\partial_{z} v_{1}\right|^{3 \theta / 4}\left|\partial_{z} v_{1}\right|^{1-\theta}\left|\partial_{x} v_{1}\right|
$$

and Hölder inequality implies that

$$
\rho \varepsilon^{2} \int_{\Omega_{T}} v_{1} \partial_{x} v_{1} \phi_{1} \leq \rho \varepsilon^{2}\left|\phi_{1}\right|_{\delta^{\prime}} C\left\|\partial_{x} v_{1}\right\|_{L^{2}\left(\Omega_{T}\right)}^{1+\theta / 4}\left\|\partial_{z} v_{1}\right\|_{L^{2}\left(\Omega_{T}\right)}^{1-\theta / 4}
$$

Now, choose $\theta$ (and thus $\delta$ ) such that $\delta^{\prime} \geq 6$. It suffices to take $\theta \leq \frac{1}{3}$, for example take $\theta=\frac{1}{3}$. Then $\delta^{\prime}=6$, and the usual Sobolev embeddings read $H^{1}(\Omega) \hookrightarrow L^{6}(\Omega)$ (which is true in dimension 2 or 3 ). Therefore, the last estimate becomes

$$
\rho \varepsilon^{2} \int_{\Omega_{T}} v_{1} \partial_{x} v_{1} \phi_{1} \leq \rho \varepsilon^{2} C\left\|\phi_{1}\right\|_{H^{1}}\left\|\partial_{x} v_{1}\right\|_{L^{2}\left(\Omega_{T}\right)}^{13 / 12}\left\|\partial_{z} v_{1}\right\|_{L^{2}\left(\Omega_{T}\right)}^{11 / 12}
$$

Now, recalling that $\left\|\partial_{z} v_{1}\right\|_{L^{2}\left(L^{2}\right)} \leq C \varepsilon$ and $\left\|\partial_{x} v_{1}\right\|_{L^{2}\left(L^{2}\right)} \leq C$, we conclude

$$
\rho \varepsilon^{2} \int_{\Omega_{T}} v_{1} \partial_{x} v_{1} \phi_{1} \leq \rho \varepsilon^{2} C\left\|\phi_{1}\right\|_{H^{1}} \varepsilon^{11 / 12}=\rho \varepsilon^{2+11 / 12} C\left\|\phi_{1}\right\|_{H^{1}} \leq C \varepsilon\left\|\phi_{1}\right\|_{H^{1}} .
$$

In a similar way, it holds

$$
\rho \varepsilon \int_{\Omega_{T}} r_{2} \partial_{z} v_{1} \phi_{1} \leq \rho \varepsilon^{2-1 / 12} C\left\|\phi_{1}\right\|_{H^{1}} \leq \widetilde{C} \varepsilon\left\|\phi_{1}\right\|_{H^{1}}
$$

For the term $\rho \varepsilon^{2} \int_{\Omega} \phi_{1}\left(v_{1}(T)-v_{1}(0)\right)$, we apply Cauchy-Schwarz inequality. $v_{1}(0)$ is bounded, and for $v_{1}(T)$, we use Poincaré inequality. It follows, using the fact that $\left|\partial_{z} v_{1}\right| \leq C \varepsilon$ :

$\rho \varepsilon^{2} \int_{\Omega} \phi_{1}\left(v_{1}(T)-v_{1}(0)\right) \leq\left(C\left|v_{1}\right|+C\right) \varepsilon^{2}\left\|\phi_{1}\right\|_{H^{1}} \leq\left(C\left|\partial_{z} v_{1}\right|+C\right) \varepsilon^{2}\left\|\phi_{1}\right\|_{H^{1}} \leq C \varepsilon^{2}\left\|\phi_{1}\right\|_{H^{1}} \leq C \varepsilon\left\|\phi_{1}\right\|_{H^{1}}$. 
For the other linear terms, a simple application of Cauchy-Schwarz inequality allows to obtain similar estimates. Indeed, it suffices to use the estimate $(5.18)$ in order to estimate the $L^{2}$-norm of $\partial_{x} v_{1}, \partial_{z} v_{1}, \tau_{11}, \tau_{12}, L_{1}, C_{1}$. For example, since $\left|\partial_{x} v_{1}\right| \leq C$, the following estimate holds:

$$
\rho \varepsilon^{2} \int_{\Omega} \partial_{x} v_{1} \partial_{x} \phi_{1} \leq \rho \varepsilon^{2}\left|\partial_{x} v_{1}\right|\left|\partial_{x} \phi_{1}\right| \leq C \varepsilon^{2}\left\|\phi_{1}\right\|_{H^{1}}
$$

For the terms $L_{1}$ and $C_{1}, C_{1}$ and the constant part of $L_{1}$ are obviously bounded uniformly in $\varepsilon$. It remains to estimate the linear term $\mathcal{L}_{1}$ of $L_{1}$. Recalling its definition and using Poincaré inequality in the second estimate:

$$
\left|\mathcal{L}_{1}\right| \leq C\left(\left|v_{1}\right|+\left|v_{2}\right|+\left|\partial_{x} v_{1}\right|+\left|\partial_{z} v_{1}\right|\right) \leq C\left(\left|\partial_{z} v_{1}\right|+\left|\partial_{x} v_{1}\right|+\left|\partial_{z} v_{2}\right|\right)
$$

Using again (5.18), the boundedness of $\mathcal{L}_{1}$ follows:

$$
\left|\mathcal{L}_{1}\right| \leq C
$$

Hence $\forall \phi_{1} \in H_{0}^{1}(\Omega)$ :

$$
\int_{\Omega} \partial_{x} \pi \phi_{1} \leq C\left(\varepsilon+\varepsilon^{2}\left|\partial_{x} v_{1}\right|+\left|\partial_{z} v_{1}\right|+\varepsilon\left|\tau_{11}\right|+\left|\tau_{12}\right|+\varepsilon^{2}\left|L_{1}\right|+\varepsilon\left|C_{1}\right|\right)\left\|\phi_{1}\right\|_{H^{1}} \leq C \varepsilon\left\|\phi_{1}\right\|_{H^{1}} .
$$

The same approach with (5.1b) gives a similar estimate, for all $\phi_{2} \in H_{0}^{1}(\Omega)$ :

$$
\int_{\Omega} \partial_{z} \pi \varphi_{2} \leq C\left(\varepsilon+\varepsilon^{4}\left|\partial_{x} v_{2}\right|+\varepsilon^{2}\left|\partial_{z} v_{2}\right|+\varepsilon^{2}\left|\tau_{12}\right|+\varepsilon\left|\tau_{22}\right|+\varepsilon^{2}\left|L_{2}\right|+\varepsilon\left|C_{2}\right|\right)\left\|\phi_{2}\right\|_{H^{1}} \leq C \varepsilon\left\|\phi_{2}\right\|_{H^{1}}
$$

Thus we can conclude that $\|\nabla \pi\|_{L^{\infty}\left(H^{-1}\right)} \leq C \varepsilon$.

Now recall that for $f \in L_{0}^{2}(\Omega)$, it holds that $|f| \leq\|\nabla q\|_{H^{-1}}$ (see for example [17]). Since $p \in L_{0}^{2}(\Omega)$ and $p^{*} \in L_{0}^{2}(\Omega), q$ lies in $L_{0}^{2}(\Omega)$. From the definition of $\pi$ as function of $q$, it is clear that $\pi \in L_{0}^{2}(\Omega)$. This allows to deduce

$$
|\pi|_{L^{\infty}\left(L^{2}\right)} \leq\|\nabla \pi\|_{L^{\infty}\left(H^{-1}\right)} \leq C \varepsilon \rightarrow 0
$$

thus $\pi$ tends to zero in $L^{\infty}\left(0, T, L_{0}^{2}(\Omega)\right)$ when $\varepsilon \rightarrow 0$. Now, since $q=\frac{\partial \pi}{\partial t}$, it follows that $q$ tends to zero in $\mathcal{D}^{\prime}\left(0, T, L_{0}^{2}(\Omega)\right)$, and therefore:

$$
\varepsilon^{2} p \underset{\varepsilon \rightarrow 0}{\rightarrow} p^{*} \quad \text { in } \mathcal{D}^{\prime}\left(0, T, L^{2}(\Omega)\right)
$$

This finishes the proof.

\subsection{Open problems}

This work concerns only the solutions of the problem (3.1) that are obtained as the limit of the regularized problem we chose (with an additional term $-\eta \Delta \boldsymbol{\sigma})$. Since there is no uniqueness result 
for problem (3.1), it is not known how other solutions behave.

Formally, the passing to the limit can be done for $a \neq 0$ (see [4]), and a similar limit problem (involving the parameter $a$, but of the same structure). However, the proof of the existence theorem in $\hat{\Omega}^{\varepsilon}$ strongly relies on the fact that $a=0$. No global results are proved in the case $a \neq 0$.

Last, since the computations are independent of the dimension of the domain $\Omega$, the result should be true in the three-dimensional case. The limit problem on $\left(\boldsymbol{u}^{*}, p^{*}, \boldsymbol{\sigma}^{*}\right)$ reads:

$$
\left\{\begin{array}{l}
(1-r) \nu \partial_{z}^{2} u_{1}^{*}-\partial_{x} p^{*}+\partial_{z} \sigma_{13}^{*}=0, \\
(1-r) \nu \partial_{z}^{2} u_{2}^{*}-\partial_{x} p^{*}+\partial_{z} \sigma_{23}^{*}=0, \\
\partial_{z} p^{*}=0 \\
\nabla \cdot \boldsymbol{u}^{*}=0 \\
-\lambda^{*} \partial_{z} u_{1}^{*} \sigma_{13}^{*}+\sigma_{11}^{*}=0 \\
-\frac{\lambda^{*}}{2} \partial_{z} u_{1}^{*} \sigma_{13}^{*}-\partial_{z} u_{2}^{*} \sigma_{23}^{*}+\sigma_{12}^{*}=0 \\
-\lambda^{*} \partial_{z} u_{2}^{*} \sigma_{23}^{*}+\sigma_{22}^{*}=0 \\
\frac{\lambda^{*}}{2} \partial_{z} u_{2}^{*}\left(\sigma_{33}^{*}-\sigma_{22}^{*}\right)-\frac{\lambda^{*}}{2} \partial_{z} u_{1}^{*} \sigma_{12}^{*}+\sigma_{23}^{*}=r \nu \partial_{z} u_{2}^{*}, \\
\lambda^{*}\left(\partial_{z} u_{1}^{*} \sigma_{13}^{*}+\partial_{z} u_{2}^{*} \sigma_{23}^{*}\right)+\sigma_{33}^{*}=0, \\
\frac{\lambda^{*}}{2} \partial_{z} u_{1}^{*}\left(\sigma_{33}^{*}-\sigma_{11}^{*}\right)-\frac{\lambda^{*}}{2} \partial_{z} u_{2}^{*} \sigma_{12}^{*}+\sigma_{12}^{*}=r \nu \partial_{z} u_{1}^{*}
\end{array}\right.
$$

\section{Acknowledgements}

The authors would like to thank P. Mironescu for useful discussions concerning section 4.3 of this paper.

\section{References}

[1] A. Assemien, G. Bayada, and M. Chambat, Inertial effects in the asymptotic behavior of a thin film flow, Asymptotic Anal. 9(3) (1994), 177-208.

[2] G. Bayada and M. Chambat, The transition between the Stokes equations and the Reynolds equation: a mathematical proof, Appl. Math. Optim. 14(1) (1986), 73-93.

[3] G. Bayada, M. Chambat, and I. Ciuperca, Asymptotic Navier-Stokes equations in a thin moving boundary domain, Asymptot. Anal. 21(2) (1999), 117-132.

[4] G. Bayada, L. Chupin, and S. Martin, Viscoelastic fluids in a thin domain, Quart. Appl. Math. 65 (2007), 625-651.

[5] G. Bayada and G. Lukaszewicz, On micropolar fluids in the theory of lubrication. Rigorous derivation of an analogue of the Reynolds equation, Internat. J. Engrg. Sci. 34(13) (1996), $1477-1490$. 
[6] Fouad Boughanim and Roland Tapiéro, Derivation of the two-dimensional Carreau law for a quasi-Newtonian fluid flow through a thin slab, Appl. Anal. 57(3-4) (1995), 243-269.

[7] A. Bourgeat, A. Mikelić, and R. Tapiéro, Dérivation des équations moyennées décrivant un écoulement non newtonien dans un domaine de faible épaisseur, C. R. Acad. Sci. Paris Sér. I Math. 316(9) (1993),965-970.

[8] F. Boyer, L. Chupin, and P. Fabrie, Numerical study of viscoelastic mixtures through a Cahn-Hilliard flow model, Eur. J. Mech. B Fluids 23(5) (2004),759-780.

[9] R. Bunoiu and S. Kesavan, Asymptotic behaviour of a Bingham fluid in thin layers, J. Math. Anal. Appl. 293(2) (2004), 405-418.

[10] L. Chupin, Some theoretical results concerning diphasic viscoelastic flows of the Oldroyd kind, Adv. Differential Equations 9(9-10) (2004), 1039-1078.

[11] E. Fernández-Cara, F. Guillén, and R. R. Ortega, Some theoretical results concerning nonNewtonian fluids of the Oldroyd kind, Ann. Scuola Norm. Sup. Pisa Cl. Sci. (4) 26 (1998), $1-29$.

[12] C. Guillopé and J.-C. Saut, Existence results for the flow of viscoelastic fluids with a differential constitutive law, Nonlinear Anal. 15(9) (1990), 849-869.

[13] P. L. Lions and N. Masmoudi, Global solutions for some Oldroyd models of non-Newtonian flows, Chinese Ann. Math. Ser. B 21(2) (2000), 131-146.

[14] E. Marušić-Paloka and M. Starčević, Rigorous justification of the Reynolds equations for gas lubrication, C. R. Mécanique 33(7) (2005), 534-541.

[15] Laetitia Paoli, Asymptotic behavior of a two fluid flow in a thin domain: from Stokes equations to Buckley-Leverett equation and Reynolds law, Asymptot. Anal. 34(2) (2003), 93-120.

[16] J.-M. Sac-Epée and K. Taous, On a wide class of nonlinear models for non-Newtonian fluids with mixed boundary conditions in thin domains, Asymptot. Anal. 44(1-2) (2005), 151-171.

[17] Roger Temam, Navier-Stokes equations, in: Studies in Mathematics and its Applications, Vol. 2, North-Holland, Amsterdam, revised edition, 1979. 